TRANSACTIONS OF THE

AMERICAN MATHEMATICAL SOCIETY

Volume 353, Number 3, Pages 853-875

S 0002-9947(00)02583-6

Article electronically published on November 8, 2000

\title{
ON THE ASYMPTOTIC GEOMETRY OF NONPOSITIVELY CURVED GRAPHMANIFOLDS
}

\author{
S. BUYALO AND V. SCHROEDER
}

\begin{abstract}
In this paper we study the Tits geometry of a 3-dimensional graphmanifold of nonpositive curvature. In particular we give an optimal upper bound for the length of nonstandard components of the Tits metric. In the special case of a $\pi / 2$-metric we determine the whole length spectrum of the nonstandard components.
\end{abstract}

\section{Introduction}

The asymptotic behavior of geodesics on a closed Riemannian manifold $M$ of nonpositive curvature may be described in terms of the boundary at infinity $\partial_{\infty} X$ of its universal covering $X$ and the action of the fundamental group $\Gamma=\pi_{1}(M)$ on $\partial_{\infty} X$. The points of $\partial_{\infty} X$ are the classes of asymptotic geodesic rays in $X$. Usually one considers two topologies on $\partial_{\infty} X$ : the standard and the metric ones. In the standard topology, points $z, z^{\prime} \in \partial_{\infty} X$ are close if they are visible from a fixed point $x \in X$ under a small angle. The metric topology is associated with the angle metric $\angle$ on $\partial_{\infty} X$, where $\angle\left(z, z^{\prime}\right)$ is defined as the supremum of the angles under which the points $z, z^{\prime} \in \partial_{\infty} X$ are visible from the points of $X$. The corresponding intrinsic metric on $\partial_{\infty} X$ is called the Tits metric, and the boundary at infinity with the metric topology is denoted by $\partial_{T} X$. The metric topology is finer than the standard one.

The group $\Gamma$ acts on $\partial_{\infty} X$ by homeomorphisms with respect to the standard topology and by isometries of the Tits metric.

A typical example of a connected subset in $\partial_{T} X$ is the boundary at infinity $\partial_{\infty} E$ of a flat $E \subset X$ of dimension $k \geq 2$, i.e. a geodesic subspace isometric to $\mathbb{R}^{k}$. In that case $\partial_{\infty} E$ is isometric to the unit sphere $S^{k-1} \subset \mathbb{R}^{k}$. Such subspaces $E$ are often associated with subgroups in $\Gamma$ isomorphic to $\mathbb{Z}^{k}$.

In other words, there are connected components in $\partial_{T} X$ whose combinatorial structure reflects the combinatorial structure of the simplicial complex $\mathcal{A}$ of (maximal) abelian subgroups in $\Gamma$, whose simplices are collections of subgroups ordered by inclusion. Such components are called standard (for precise definition see $\S 2$ ).

At the same time, there are nonstandard components in $\partial_{T} X$, i.e. such that any point of them is not a boundary point of any $k$-flat in $X$ with $k \geq 2$.

The very existence of nondegenerate (i.e. different from a point) nonstandard components in $\partial_{T} X$ is not obvious, and the first examples appeared only recently

Received by the editors July 28, 1997 and, in revised form, May 5, 1999.

2000 Mathematics Subject Classification. Primary 53C20.

The first author was supported by RFFI Grant 96-01-00674 and CRDF Grant RM1-169.

The second author was supported by the Swiss National Science Foundation. 
(see [CK], [HS]; in 1992 in a conversation with the first author, B. Leeb had mentioned a possibility of existence of fat triangles in a graphmanifold, which are not contained in a block, this is equivalent to the existence of nonstandard components; in 1996 C. Croke and B. Kleiner gave the first examples of nondegenerate nonstandard components by constructing certain nonsmooth metrics of nonpositive curvature on graphmanifolds).

Nonstandard connected components of $\partial_{T} X$ are the main subject of the present work. Here we restrict to the case of 3-dimensional graphmanifolds, i.e. the simplest case, where nonstandard components exist. For a graphmanifold $M$ with nonpositively curved metric every nonstandard component is a segment of length $<\pi$ (see Proposition 2.12).

Our first result gives an optimal estimate for the length of a nonstandard component from above.

0.1. Theorem. For a nonpositively curved metric $g$ on a graphmanifold $M$ the length of any nonstandard component $\mathcal{I}_{w}$ of $\partial_{T} X$ satisfies

$$
L_{g}(w) \leq \omega(g),
$$

where $\omega(g) \in[\pi / 2, \pi)$ is the maximal angle between singular directions of adjacent blocks of $M$.

Next, we show that there exists a component $\mathcal{I}_{w}$ of the maximal length $L_{g}(w)=$ $\omega(g)$. Moreover, we obtain a slightly stronger result bringing primitive components into the game. A primitive nonstandard component $\mathcal{I}_{w}$ is associated with a gluing torus $T_{u}$ : every geodesic ray $c$ in $X, c(\infty) \in \mathcal{I}_{w}$ descends to a geodesic $\bar{c}$ in $M$, which starting from some moment lives only in two adjacent maximal blocks of $M$ skipping from one to another through $T_{u}$.

0.2. Theorem. For any gluing torus $T_{u} \subset M$ there exists an associated primitive component $\mathcal{I}_{w} \subset \partial_{T} X$ of the maximal length $L_{g}(w)=\omega_{u}(g)$, where $\omega_{u}(g) \in[\pi / 2, \pi)$ is the maximal angle between singular directions on $T_{u}$.

The set of nonstandard components $\mathcal{W}$ is independent of the choice of the nonpositively curved metric and defined only by the fundamental group (see §2). The metric defines only lengths $L(w), w \in \mathcal{W}$ of the components $\mathcal{I}_{w} \subset \partial_{T} X$.

0.3. Corollary. If metrics $g_{0}, g_{1}$ of nonpositive curvature on a graphmanifold $M$ have the same marked length spectrum $\left(\mathcal{W}, L_{g_{0}}\right)=\left(\mathcal{W}, L_{g_{1}}\right)$ of nonstandard connected components of the Tits boundaries $\partial_{T} X_{0}, \partial_{T} X_{1}$, then $\partial_{T} X_{0}$ and $\partial_{T} X_{1}$ are $\Gamma$-equivariantly isometric, $\Gamma=\pi_{1}(M)$.

In the case $\omega_{u}(g)=\pi / 2$ we have a much better understanding of the length spectrum for the associated primitive components.

0.4. Theorem. Assume that the angle between the singular directions of a gluing torus $T_{u} \subset M$ is $\omega_{u}=\omega_{u}(g)=\pi / 2$. Then the length spectrum of the associated primitive components $\mathcal{I}_{w}, w \in \mathcal{W}_{u}$ of $\partial_{T} X$ coincides with $\left[0, \omega_{u}\right]$.

For the definition of graphmanifolds and the notions mentioned in the results above see $\S 1$. In this work we consider $C^{1}$-smooth metrics on $M$. The condition for a metric to be nonpositively curved is understood is the sense of Alexandrov, i.e. in the sense of the angle comparison. Probably, our results are true for general nonpositively curved metrics on graphmanifolds. 
Theorem 0.4 is motivated by the question, what is the role of nonstandard components in the asymptotic geometry of nonpositively curved metrics? In particular, by the questions, how does the marked length spectrum $L_{g}(w), w \in \mathcal{W}$ of nonstandard components depend on the metric $g$, and in which degree this spectrum defines the metric itself?

Metrics of nonpositive curvature on graphmanifolds are typical examples of metrics of rank 1 in the sense of $[\mathrm{BBE}]$. Here the situation is drastically distinct from that which takes place for the spaces of rank $\geq 2$ or hyperbolic spaces. While for higher rank spaces there are no nontrivial metric deformations in the class of nonpositive curvature, in the hyperbolic case it is impossible to change the equivariant topology on $\partial_{\infty} X$ by changing the metric in the class considered. In the rank one case there are, in general, metric deformations but the asymptotic geometry is very sensitive to the change of the metric.

There are two types of deformations $g_{t}$ of nonpositively curved metrics on a graphmanifold $M$. First, there are rigid type deformations, when the angle between $S^{1}$-factors of some adjacent blocks is changed (see $\S 1$ ) and, correspondingly, the geometry of the principal connected component $\mathcal{F}$ (see $\S 2$ ) of $\partial_{T} X$ is changed. In $[\mathrm{Bu}$, an example of the rigid type deformation is given. We show (see Theorem 2.10) that any change of the geometry of $\mathcal{F}$ under the metric change $g_{0} \mapsto g_{1}$ always leads to the result that there is no continuous $\Gamma$-equivariant map $\partial_{\infty} X_{0} \rightarrow \partial_{\infty} X_{1}$, where $\Gamma=\pi_{1}(M)$ (recall that the spaces $\partial_{\infty} X_{0}, \partial_{\infty} X_{1}$ are homeomorphic to the sphere $S^{2}$ ).

Second, a metric deformation $g_{t}$ might appear in the soft type, when the geometry of the principal component $\mathcal{F}$ is kept the same. Such deformations are, for example, all deformations in the class of $\pi / 2$-metrics.

If the angle between the singular directions $\omega_{u}(g)=\pi / 2$ for every gluing torus $T_{u}$ in $M$, then the metric $g$ is called a $\pi / 2$-metric (see $\S 1$ for the precise definition). Not every graphmanifold admitting a nonpositively curved metric, carries a $\pi / 2$ metric. At the same time, there are graphmanifolds on which any nonpositively curved metric is a $\pi / 2$-metric. In $\S 1$ we indicate necessary and sufficient conditions for a graphmanifold to possess a $\pi / 2$-metric.

The change of the equivariant topology $\left(\partial_{\infty} X, \Gamma\right)$ in the soft type deformation is not so obvious, however, it also takes place. A corresponding example was given by C. Croke and B. Kleiner. This happens because some (nondegenerate) nonstandard components degenerate even in the soft type deformation. Probably, the behavior of the length spectrum of nonstandard components is highly sensitive to any (nontrivial) metric deformation.

Structure of the paper. $\S 1$ contains some background material on graphmanifolds and metrics of nonpositive curvature on them. In particular, we give there a necessary and sufficient condition for a graphmanifold to carry a $\pi / 2$-metric.

In $\S 2$, after recalling the definitions of the standard topology and Tits metric on $\partial_{\infty} X$, we describe the decomposition of the Tits boundary $\partial_{T} X$ for a nonpositively curved graphmanifold into connected components, showing that this decomposition can defined by the fundamental group $\Gamma$. Next, we show that each nonprincipal connected component of $\partial_{T} X$ is an interval of length $<\pi$ and may be degenerate (Proposition 2.12). We also discuss here the question of how a deformation of a metric effects the $\Gamma$-equivariant topology of $\partial_{\infty} X$ (Theorem 2.10 and Proposition 2.11). 
Theorems $0.1,0.2$ and 0.4 are proved in $\S 3$.

Acknowledgment. We thank Chris Croke for explaining to us the examples of [CK]. The first author is grateful to the University of Zürich for the support and hospitality while working on this paper.

\section{Preliminaries on metrics of nOnPositive CURVATURE ON GRAPHMANIFOLDS}

Here we collect necessary facts about nonpositively curved metrics on graphmanifolds. The proofs can be found in or extracted from [BK1, BB]. For general reference we also refer to $\mathrm{BGS}$, and the books of Ballmann [B] and Eberlein [E].

1.1. In the present work a graphmanifold is understood as a closed 3 -dimensional manifold $M$, for which there exists a nonempty minimal collection $\mathcal{E}$ of disjointly embedded incompressible tori and Klein bottles such that the abstract completion $M_{v}$ of each connected component of the complement to $\mathcal{E}$ is a Seifert fiber space. The manifold $M_{v}$ is called a maximal block of $M$. Furthermore, we assume that each maximal block admits a geometric structure of type $H^{2} \times \mathbb{R}$, i.e. a Riemannian metric such that the universal covering $\widetilde{M}_{v}$ is isometric to the metric product $Y_{v} \times \mathbb{R}$, where $Y_{v} \subset H^{2}$ is a closed convex subset bounded by infinite geodesics.

Any finite covering $M^{\prime}$ for $M$ is also a graphmanifold of that type. It is known (see $[\mathrm{Ka}]$ ) that there exists a finite cover $M^{\prime} \rightarrow M$ such that every maximal block $M_{v}^{\prime} \subset M^{\prime}$ is homeomorphic to $F_{v}^{\prime} \times S^{1}$, where $F_{v}^{\prime}$ is a compact surface with nonempty boundary. In that case one can assume that $M^{\prime}$ and, consequently, all $F_{v}^{\prime}$ are orientable. The condition of the existence of a $H^{2} \times \mathbb{R}$ structure excludes the possibility for $F_{v}^{\prime}$ to be the disc or the annulus.

Since the asymptotic geometry of $M$ does not change, while taking a finite covering, in the sequel we assume for simplicity that $M$ is orientable and every maximal block $M_{v}$ is homeomorphic to $F_{v} \times S^{1}$, where $F_{v}$ is a compact orientable surface with nonempty boundary different from the disc and the annulus. In that case the separating collection $\mathcal{E}$ consists of tori.

1.2. In the present work, we consider $C^{1}$-smooth Riemannian metrics of nonpositive curvature on $M$. An important example of such a metric is a geometrization of $M$, i.e. a metric which induces on every maximal block a geometric structure of type $H^{2} \times \mathbb{R}$. A geometrization exists iff the graphmanifold possesses a nonpositively curved metric (see [Le]). Let $g$ be such a metric. Then each torus $T$ of the collection $\mathcal{E}$ can be chosen flat and geodesic. The curves on $T$ representing the factor $S^{1}$ from the decomposition $M_{v_{i}}=F_{v_{i}} \times S^{1}, i=0,1$ for two blocks adjacent along $T$ are closed geodesics representing (due to the minimality of $\mathcal{E}$ ) independent elements of the homology group $H_{1}(T ; \mathbb{Z}) \simeq \mathbb{Z}^{2}$. Thus, if an orientation of the factors $S^{1}$ is fixed, the angle $\omega$ between these geodesics is well defined, $0<\omega<\pi$. The metric $g$ is said to be a $\pi / 2$-metric, if $\omega=\pi / 2$ for all gluing tori $T \in \mathcal{E}$.

1.3. Here we give a necessary and sufficient condition for a graphmanifold $M$ to possess a $\pi / 2$-metric. It is formulated in terms of topological invariants of $M$ introduced in BK1, BK2 and called charges.

Let $V$ be the set of all maximal blocks of $M$. For $v \in V$ let $\partial v$ be the set of the boundary components of the block $M_{v}, U=\bigcup_{v \in V} \partial v . V$ is the vertex set of the graph $G=G_{M}$ of $M$, whose set of oriented edges is $U$. A vertex $v \in V$ is initial 
for an edge $u \in U$ if and only if $u \in \partial v$. The set of gluing tori $\mathcal{E}$ can be identified with the set of nonoriented edges of $G$, i.e. pairs $(u,-u), u \in U$.

To define charges $k_{v}, v \in V$ we fix an orientation of $M$. This defines an orientation of every block $M_{v}$, for which we also fix an orientation of the factor $S^{1}$ in the decomposition $M_{v}=F_{v} \times S^{1}$.

For $u \in \partial v$ let $L_{u} \simeq \mathbb{Z}^{2}$ be the homology group $H_{1}\left(T_{u} ; \mathbb{Z}\right)$ of the torus $T_{u}=$ $\left(\partial M_{v}\right)_{u}$. Next we choose a basis $\left\{\left(z_{u}, f_{u}\right) \mid u \in \partial v\right\}$ of the group $H_{1}\left(\partial M_{v} ; \mathbb{Z}\right)=$ $\bigoplus_{u \in \partial v} L_{u}$ such that the basis $\left(z_{u}, f_{u}\right)$ of the lattice $L_{u}$ is compatible with the orientation induced on $\partial M_{v}$, the element $f_{u}$ represents the oriented factor $S^{1}$ of the block $M_{v}$, and the sum $\bigoplus_{u \in \partial v} z_{u}$ lies in the kernel of the inclusion homomorphism

$$
H_{1}\left(\partial M_{v} ; \mathbb{Z}\right) \rightarrow H_{1}\left(M_{v} ; \mathbb{Z}\right),
$$

i.e. represents the boundary $\partial F_{v}$ of the surface $F_{v}$ from the decomposition $M_{v}=$ $F_{v} \times S^{1}$. The collection $(z, f)=\left\{\left(z_{u}, f_{u}\right) \mid u \in \partial v, v \in V\right\}$ is called a Waldhausen basis. In general, the choice of a Waldhausen basis is not unique even when the orientations as above are fixed.

To each oriented edge $u$ of the graph $G$, there is a corresponding gluing map of boundary components of adjacent blocks, which induces an isomorphism $g_{u}$ : $L_{-u} \rightarrow L_{u}$; with respect to the chosen bases, $g_{u}$ has the matrix

$$
\left(\begin{array}{ll}
a_{u} & b_{u} \\
c_{u} & d_{u}
\end{array}\right) \in G L(2, \mathbb{Z})
$$

i.e.

$$
\begin{gathered}
g_{u}\left(z_{-u}\right)=a_{u} z_{u}+c_{u} f_{u}, \\
g_{u}\left(f_{-u}\right)=b_{u} z_{u}+d_{u} f_{u} .
\end{gathered}
$$

We have $\operatorname{det} g_{u}=a_{u} d_{u}-b_{u} c_{u}=-1$ since $M$ is orientable, and $g_{-u}=g_{u}^{-1}, b_{-u}=$ $b_{u} \neq 0$ because the elements $f_{u}$ and $g_{u}\left(f_{-u}\right)$ representing the factors $S^{1}$ of adjacent blocks are independent.

The charge of a vertex $v \in V$ is defined as

$$
k_{v}=\sum_{u \in \partial v} d_{u} / b_{u}
$$

and is an invariant of the oriented manifold $M$, i.e. it does not depend on the choice of orientations of factors $S^{1}$ and the Waldhausen basis. Charges change sign if the orientation of $M$ is changed (see [BK1]).

1.4. Theorem. A graphmanifold $M$ admits a $\pi / 2$-metric if and only if $k_{v}=0$ for all $v \in V$.

This fact easily follows from the decomposition principle (see [BK1 §13]). Let us give several examples illustrating Theorem 1.4.

1.5. Examples. 1. Assume that the graph of $M$ consists of two vertices $v_{0}, v_{1}$ connected by an edge $(u,-u)$. The gluing map is given by the matrix

$$
g_{u}=\left(\begin{array}{ll}
a & b \\
c & d
\end{array}\right)
$$

which might be an arbitrary element of $G L(2, \mathbb{Z})$ with $\operatorname{det} g_{u}=-1$ and $b \neq 0$. Then for the charges of vertices we have $k_{0}=d / b, k_{1}=-a / b$. The manifold $M$ admits a nonpositively curved metric iff $k_{0}=k_{1}=0$. This is equivalent to the condition $a=d=0, b=c= \pm 1$ (see [Le, [BK1]). Notice that for such $M$ there is only 
one Waldhausen basis, provided an orientation of $M$ and orientations of the block fibers are fixed. Any nonpositively curved metric on $M$ is a $\pi / 2$-metric.

2. Assume that the graph of $M$ consists of one vertex $v$ and one edge $(u,-u)$, which is therefore a loop. As in the previous example, there is only one gluing map given by the matrix

$$
g_{u}=\left(\begin{array}{ll}
a & b \\
c & d
\end{array}\right) \in G L(2, \mathbb{Z})
$$

(this time the choice of Waldhausen bases is not unique). Then $k_{v}=(d-a) / b$. The manifold $M$ admits a nonpositively curved metric iff $|d-a|<2$. There is a $\pi / 2$-metric on $M$ iff $d=a$. In the last case any nonpositively curved metric on $M$ is a $\pi / 2$-metric. For $a=c=d=1, b=2$ we obtain an example of $M$, on which every nonpositively curved metric is a $\pi / 2$-metric and which cannot be obtained by the switching generators gluing. This is because the number $|b|$ is a topological invariant of $M$ (an intersection index, see [BK1]), and it is equal to 1, if the generators switch.

3. Assume that the graph of $M$ is a circle with an odd number $|V| \geq 1$ of edges, and $k_{v}=0$ for all $v \in V$. Then any nonpositively curved metric on $M$ is a $\pi / 2$-metric. In particular, every deformation in the class of nonpositively curved metrics on $M$ is soft (and there are plenty of such nontrivial deformations).

4. Assume that the graph of $M$ is a circle with an even number $|V| \geq 2$ of edges, and $k_{v}=0$ for all $v \in V$. Then $M$ possesses $\pi / 2$-metrics as well as non- $\pi / 2$-metrics of nonpositive curvature, for which the angle $\omega$ between singular directions of some adjacent blocks can take any value from the interval $(0, \pi)$. In particular, there are nontrivial rigid deformations in the class of nonpositively curved metrics. In the simplest case $|V|=2$ the angles $\omega_{0}, \omega_{1}$ for the corresponding edges $u_{0}, u_{1}$ are related by

$$
\cos \omega_{0}+\cos \omega_{1}=0
$$

if $b_{0}=b_{1}$, and a rigid deformation can be described with a parameter $\phi \in[0, \pi / 2)$ as $\omega_{0}=\pi / 2-\phi, \omega_{1}=\pi / 2+\phi$; see $[\mathrm{Bu}]$.

\section{ThE BOUNDARY AT INFINITY OF A GRAPHMANIFOLD}

2.1. Let $X$ be a Hadamard space, i.e. a complete simply connected metric space of nonpositive curvature. Geodesic rays $c, c^{\prime}:[0, \infty) \rightarrow X$ are called asymptotic, if the distances $\left|c(t) c^{\prime}(t)\right|$ are bounded as $t \rightarrow \infty$ (here and in the sequel we always assume that geodesic rays are parametrized by arc length). The boundary at infinity $\partial_{\infty} X$ of $X$ consists of classes of asymptotic geodesic rays in $X$. Recall the definitions of the standard topology and the Tits metric on $\partial_{\infty} X$.

2.1.1. Fix a point $x_{0} \in X$. Then any point $z \in \partial_{\infty} X$ can be identified with the geodesic ray $z:[0, \infty) \rightarrow X, z(0)=x_{0}, z(\infty)=z$. A base of the standard topology consists of the sets

$$
U_{x_{0}, t}(z)=\left\{z^{\prime} \in \partial_{\infty} X|| z(t) z^{\prime}(t) \mid<1\right\}, \quad z \in \partial_{\infty} X, t>0 .
$$

2.1.2. For $z, z^{\prime} \in \partial_{\infty} X$ and $t, t^{\prime}>0$ let $\bar{x}_{0} \bar{z}(t) \bar{z}^{\prime}\left(t^{\prime}\right) \subset \mathbb{R}^{2}$ be the comparison triangle for the triangle $x_{0} z(t) z^{\prime}\left(t^{\prime}\right) \subset X$. Then the limit

$$
\angle\left(z, z^{\prime}\right)=\lim _{t, t^{\prime} \rightarrow \infty} \angle \bar{z}(t) \bar{x}_{0} \bar{z}^{\prime}\left(t^{\prime}\right)
$$


exists and is independent of the choice of $x_{0} \in X$. This defines the angle metric $\angle\left(z, z^{\prime}\right) \in[0, \pi]$ on $\partial_{\infty} X$. The corresponding intrinsic metric is called the Tits metric on $\partial_{\infty} X$. We use the notation $\partial_{\infty} X$ for the boundary at infinity with the standard topology and $\partial_{T} X$ for the boundary at infinity with the Tits metric.

2.2. From now on we assume that $X$ is a universal metric covering of a graphmanifold $M=\bigcup_{v \in V} M_{v}$ with a nonpositively curved metric $g$. Then $X=\bigcup_{\alpha \in \mathcal{A}} X_{\alpha}$, where $X_{\alpha}$ is a universal covering of some maximal block $M_{v}=F_{v} \times S^{1}$. The set $X_{\alpha}$ is also called $a$ block of $X$.

2.2.1. Every block $X_{\alpha}, \alpha \in \mathcal{A}$ is a closed convex subset in $X$ isometric to the metric product $Y_{\alpha} \times \mathbb{R}$, where the surface $Y_{\alpha}$ is a universal covering of $F_{\alpha}$ with a nonpositively curved metric and a geodesic boundary. In particular, every connected component of $\partial X_{\alpha}$ is a 2-flat in $X$ (covering some gluing torus in $M$ ). If different blocks $X_{\alpha}, X_{\alpha^{\prime}}$ intersect, then their intersection $X_{\alpha} \cap X_{\alpha^{\prime}}$ is the common boundary component of these blocks, i.e. a flat in $X$.

2.2.2. The surface $Y_{\alpha}$ is cocompact, thus its boundary components are pairwise separated by a distance $\geq \rho$, where $\rho>0$ depends only on the metric $g$ and is independent of $\alpha \in \mathcal{A}$, because the manifold $M$ consists of a finite number of maximal blocks $M_{v}$.

2.2.3. The metric on $Y_{\alpha}$ is hyperbolic in the sense of Gromov, i.e. for some $\delta>0$ each side of any triangle in $Y_{\alpha}$ lies in a $\delta$-neighborhood of the union of two other sides. Again, $\delta=\delta(g)$ depends only on the metric $g$ and is independent of $\alpha \in \mathcal{A}$.

The last two properties of the surfaces $Y_{\alpha}$ will systematically be used in the sequel.

2.2.4. For instance, it follows from 2.2 .2 that width $\left(X_{\alpha}\right) \geq \rho$ for all $\alpha \in \mathcal{A}$, where width $\left(X_{\alpha}\right)<\infty$ is the shortest distance in $X$ between the points of different components of $\partial X_{\alpha}$.

2.2.5. Lemma. There exists a constant $\rho_{1}>0$, which depends only on the metric $g$ and is independent of $\alpha \in \mathcal{A}$ such that the following is true.

If geodesic segments $x y, x^{\prime} y^{\prime} \subset Y_{\alpha}$ connect a boundary component $C \subset \partial Y_{\alpha}(x$, $\left.x^{\prime} \in C\right)$ with components $D, D^{\prime} \subset \partial Y_{\alpha}, \angle y x x^{\prime}+\angle y^{\prime} x^{\prime} x \geq \pi$ and $\left|x x^{\prime}\right| \geq \rho_{1}$, then $D \neq D^{\prime}$.

Proof. One can take as $\rho_{1}$ the minimal displacement of a nontrivial isometry $\gamma \in$ $\Gamma_{v}=\pi_{1}\left(F_{v}\right)$, which leaves $C$ invariant. Here $\Gamma_{v}$ is the deck transformation group of the covering $Y_{\alpha} \rightarrow F_{v}$. Since $C$ is the unique boundary component of $Y_{\alpha}$, invariant for $\gamma$, the claim follows.

We shall refer to this lemma as the periodicity argument.

2.2.6. The boundary at infinity $\partial_{\infty} X_{\alpha}$ is the suspension over the Cantor set $\partial_{\infty} Y_{\alpha}$, and the space $\partial_{T} X_{\alpha}$ is a graph with two vertices corresponding to the ends of the factor $\mathbb{R}$ and connected by edges of length $\pi$. These edges one-to-one correspond to the points of $\partial_{\infty} Y_{\alpha}$. In particular, $\partial_{T} X_{\alpha}$ is connected and has diameter equal to $\pi$.

Since $X_{\alpha} \subset X$ is convex, we have $\partial_{\infty} X_{\alpha} \subset \partial_{\infty} X, \partial_{T} X_{\alpha} \subset \partial_{T} X$. 
The vertices of the graphs $\partial_{T} X_{\alpha}$ are called singular points of $\partial_{T} X$. Every singular point $z \in \partial_{T} X$ uniquely defines the corresponding block $X_{\alpha}, \alpha=\alpha(z)$, and $\alpha(-z)=\alpha$, where $-z$ is the opposite to $z$ vertex of the graph $\partial_{T} X_{\alpha}$.

2.2.7. Let $\alpha, \alpha^{\prime} \in \partial_{T} X$ be singular points, for which the blocks $X_{\alpha}, X_{\alpha^{\prime}}$ are adjacent, $M_{v}, M_{v^{\prime}}$ the corresponding maximal blocks of $M$. The points $\alpha, \alpha^{\prime}$ represent the oriented $S^{1}$-factors of $M_{v}, M_{v^{\prime}}$, and $\omega=\angle\left(\alpha, \alpha^{\prime}\right), 0<\omega<\pi$ is the angle between the last on the gluing torus $T$, which is covered by $X_{\alpha} \cap X_{\alpha^{\prime}}$.

\section{Encoding of the connected components of $\partial_{T} X$}

2.3. Let $W$ be the set of infinite (in one direction) strings $w$ consisting of letters of the alphabet $\mathcal{A}$; we require

(a) any letter $\alpha \in \mathcal{A}$ enters $w$ at most one time;

(b) if letters $\alpha, \alpha^{\prime} \in w$ are neighboring, then $X_{\alpha} \cap X_{\alpha^{\prime}}$ is a flat in $X$ for the corresponding blocks $X_{\alpha}, X_{\alpha^{\prime}}$.

The strings $w, w^{\prime} \in W$ are equivalent if they have a common tail; notation: $w \sim w^{\prime}, \mathcal{W}=W / \sim$.

Any geodesic ray $c:[0, \infty) \rightarrow X$ defines, obviously, a string (finite or infinite) $w_{c}$ of letters of $\mathcal{A}$.

2.3.1. Lemma. For asymptotic rays $c, c^{\prime} \in z \in \partial_{\infty} X$ we have

(i) $w_{c}$ is finite if and only if $w_{c^{\prime}}$ is finite;

(ii) if $w_{c}, w_{c^{\prime}}$ are infinite, then $w_{c} \sim w_{c^{\prime}}$.

Proof. (i) If $w_{c}$ is finite, then we can assume that $w_{c}=\{\alpha\}$ consists of one letter $\alpha \in \mathcal{A}$, i.e. $c \subset X_{\alpha}$. If $w_{c^{\prime}}$ is infinite, then $\operatorname{dist}\left(X_{\alpha}, X_{\alpha^{\prime}}\right) \rightarrow \infty$ as $\alpha^{\prime} \rightarrow \infty$, $\alpha^{\prime} \in w_{c^{\prime}}$, because the number of blocks between $X_{\alpha}$ and $X_{\alpha^{\prime}}$ tends to infinity, while width $\left(X_{\beta}\right) \geq \rho>0$ for any $\beta \in \mathcal{A}$. Thus dist $\left(c^{\prime}(t), c\right) \rightarrow \infty$ as $t \rightarrow \infty$. This contradicts the assumption that the rays $c, c^{\prime}$ are asymptoptic.

(ii) If the strings $w_{c}, w_{c^{\prime}} \in W$ are not equivalent, then, obviously, some tails of them have no common letter. It follows that $\operatorname{dist}\left(X_{\alpha}, X_{\alpha^{\prime}}\right) \rightarrow \infty$ as $\alpha, \alpha^{\prime} \rightarrow \infty$, $\alpha \in w_{c}, \alpha^{\prime} \in w_{c^{\prime}}$. Furthermore,

$$
\operatorname{dist}\left(\bigcup_{\alpha \in w_{c}} X_{\alpha}, X_{\alpha^{\prime}}\right) \rightarrow \infty \quad \text { as } \quad \alpha^{\prime} \rightarrow \infty .
$$

On the other hand, $c \subset \bigcup_{\alpha \in w_{c}} X_{\alpha}$, hence dist $\left(c^{\prime}(t), c\right) \rightarrow \infty$ as $t \rightarrow \infty$, a contradiction.

2.3.2. It follows from Lemma 2.3.1 that the set $\partial_{\infty} X$ is a disjoint union of two subsets $\mathcal{F}$ and $\mathcal{I}$, where

$z \in \mathcal{F}$ iff for any ray $c \in z$ the string $w_{c}$ is finite;

$z \in \mathcal{I}$ iff for any ray $c \in z$ the string $w_{c}$ is infinite.

2.3.3. On the structure of $\mathcal{F}$. This set coincides with the union

$$
\mathcal{F}=\bigcup_{\alpha \in \mathcal{A}} \partial_{\infty} X_{\alpha}
$$

Indeed, for any point $z \in \partial_{\infty} X_{\alpha}$ there is a ray $c \in z$ with $w_{c}=\{\alpha\}$, thus $z \in \mathcal{F}$. Conversely, for $z \in \mathcal{F}$ any ray $c \in z$ generates a finite string $w_{c}$. Thus there is a subray $c^{\prime} \subset c, c^{\prime} \in z$ with $w_{c^{\prime}}=\{\alpha\}$. Hence $z \in \partial_{\infty} X_{\alpha}$ for some $\alpha \in \mathcal{A}$.

In particular, $\mathcal{F} \subset \partial_{T} X$ is a connected subset, because $\bigcup_{\alpha \in \mathcal{A}} \partial_{\infty} X_{\alpha}$ is connected. 
2.4. By Lemma 2.3.1, there is a map $f: \mathcal{I} \rightarrow \mathcal{W}$, where $f(z)$ is the class of the string $w_{c}$ for a ray $c \in z$.

2.4.1. Lemma. The map $f$ is surjective.

Proof. For a string $w \in W$ let us consider a sequence $x: \mathbb{N} \rightarrow X, x(n) \in X_{\alpha_{n}}$, where $w=\alpha_{1} \alpha_{2} \ldots$ Then the sequence of geodesic segments $x(1) x(n)$ subconverges to a ray $c:[0, \infty) \rightarrow X$, because $|x(1) x(n)| \rightarrow \infty$ as $n \rightarrow \infty$ by the same argument as above. We claim that $w_{c}=w$. It suffices for the proof of the lemma, because then $f([c])=[w]$.

The set $\bigcup_{\alpha \in w} X_{\alpha}=: X_{w} \subset X$ is closed and convex. Thus $c \subset X_{w}$, and it suffices to show that the string $w_{c}$ is infinite.

Assume that it is not the case, and let $\alpha \in w$ be the last letter. For the flat $E_{2}=X_{\alpha+2} \cap X_{\alpha+3}$ its boundary at infinity $\partial_{\infty} E_{2}$ does not intersect $\partial_{\infty} E_{0}$ for $E_{0}=X_{\alpha} \cap X_{\alpha+1}$. Thus for a point $x \in E_{2}$ the ray $c^{\prime}:[0, \infty) \rightarrow X, c^{\prime}(0)=x$, asymptotic to $c$ forms an angle $\geq \phi>0$ with any vector from $T_{x} X$ tangent to $E_{2}$ (recall, we assume that the metric $g$ is $C^{1}$-smooth). But then the segments $x x_{\alpha+k}$ for $k \geq 3$ have angles $\geq \phi$ with $c^{\prime}$ and thus cannot converge to it, a contradiction.

2.4.2. In fact, it is proven that if $z \in \partial_{\infty} X$ is a limit of a sequence $x(n) \in X_{\alpha_{n}}$, where $w=\alpha_{1} \alpha_{2} \cdots \in W$, then $z \in \mathcal{I}$ and $f(z)=[w]$.

2.5. For $w \in \mathcal{W}$ we denote by $\mathcal{I}_{w}=\{z \in \mathcal{I} \mid f(z)=w\}$. By Lemma 2.4.1, the set $\mathcal{I}_{w} \subset \partial_{\infty} X$ is nonempty, and by Lemma 2.3.1(ii) we have

$$
\mathcal{I}=\bigcup_{w \in \mathcal{W}} \mathcal{I}_{w}
$$

So, we have a decomposition (disjoint union)

$$
\partial_{\infty} X=\mathcal{F} \cup \bigcup_{w \in \mathcal{W}} \mathcal{I}_{w}
$$

no one element of which is nonempty. Furthermore, $\mathcal{F} \subset \partial_{T} X$ is connected.

It will be shown that this decomposition is exactly the decomposition of the Tits boundary into its connected components (see Corollary 2.9).

It follows from 2.4.2 that if a sequence $\left\{z_{n}\right\} \subset \mathcal{I}_{w}$ converges to some point $z \in \partial_{\infty} X$ with respect to the standard topology, then $z \in \mathcal{I}_{w}$, i.e. $\mathcal{I}_{w}$ is closed both in the standard and the metric topologies.

2.6. Proposition. Each set $\mathcal{I}_{w} \subset \partial_{T} X, w \in \mathcal{W}$ is connected and has diameter $<\pi$.

Proof. The first assertion follows from the second one. For $z, z^{\prime} \in \mathcal{I}_{w}$ we consider rays $c \in z, c^{\prime} \in z^{\prime}$ with a common vertex $x$. By the definition of $\mathcal{I}_{w}$ we have that $w_{c}=w_{c^{\prime}}$ represent the same class $w \in W$.

Assume that the Proposition is not true. Then for any $\epsilon>0$ there are $z, z^{\prime} \in \mathcal{I}_{w}$ and a point $x \in X$ with $\angle_{x}\left(z, z^{\prime}\right)>\pi-\epsilon$.

For $\alpha \in w_{c}$ let $c_{\alpha}=c \cap X_{\alpha}, c_{\alpha}^{\prime}=c^{\prime} \cap X_{\alpha}, \bar{c}_{\alpha}, \bar{c}_{\alpha}^{\prime} \subset Y_{\alpha}$ be the projections of segments $c_{\alpha}, c_{\alpha}^{\prime}$ on the factor $Y_{\alpha}$ from the decomposition $X_{\alpha}=Y_{\alpha} \times \mathbb{R}$. We represent the segments $c_{\alpha}, c_{\alpha}^{\prime}$ as $c_{\alpha}=x_{\alpha} y_{\alpha}, c_{\alpha}^{\prime}=x_{\alpha}^{\prime} y_{\alpha}^{\prime}$, where $x_{\alpha}, x_{\alpha}^{\prime}$ are the points where the rays $c, c^{\prime}$ enter the block $X_{\alpha}$, and $y_{\alpha}, y_{\alpha}^{\prime}$ are the points where $c$, $c^{\prime}$ leave $X_{\alpha}$.

By the assumption, $x_{\alpha}, x_{\alpha}^{\prime}$ lies in the same connected component of the boundary $\partial X_{\alpha}$. Since $\angle_{x}\left(z, z^{\prime}\right)>\pi-\epsilon$, the angles at the vertices $x_{\alpha}, x_{\alpha}^{\prime}$ of the triangle $x x_{\alpha} x_{\alpha}^{\prime}$ 
are small, their sum $<\epsilon$. Thus the directions of $c, c^{\prime}$ at $x_{\alpha}, x_{\alpha}^{\prime}$ are almost opposite and close to the corresponding opposite directions of $x_{\alpha} x_{\alpha}^{\prime}$. It is also clear that $\left|x_{\alpha} x_{\alpha}^{\prime}\right| \rightarrow \infty$ as $\alpha \rightarrow \infty, \alpha \in w_{c}$. If the direction of $x_{\alpha} x_{\alpha}^{\prime}$ forms the angle $\geq \epsilon_{0}>\epsilon$ with the factor $\mathbb{R}=\mathbb{R}_{\alpha}$ in its component of $\partial X_{\alpha}$, then $\left|\bar{x}_{\alpha} \bar{x}_{\alpha}^{\prime}\right| \rightarrow \infty$ as $\alpha \rightarrow \infty$, and the segments $\bar{c}_{\alpha}, \bar{c}_{\alpha}^{\prime}$ form the angles close to $\pi$ with $\bar{x}_{\alpha} \bar{x}_{\alpha}^{\prime} \subset \partial Y_{\alpha}$. Now the periodicity argument (Lemma 2.2.5) shows that the segments $\bar{c}_{\alpha}, \bar{c}_{\alpha}^{\prime}$ cannot end in the same component of $\partial Y_{\alpha}$, a contradiction.

Thus the direction of $x_{\alpha} x_{\alpha}^{\prime}$ is $\epsilon$-close to the direction of $\mathbb{R}_{\alpha}$. Let $\underline{x}_{\alpha}, \underline{x}_{\alpha}^{\prime}, \underline{y}_{\alpha}, \underline{y}_{\alpha}^{\prime}$ be the projections on $\mathbb{R}_{\alpha}$ (in its components of $\partial X_{\alpha}$ ), $T_{\alpha}=\left|c_{\alpha}\right|, T_{\alpha}^{\prime}=\left|c_{\alpha}^{\prime}\right|$ the life times of the rays $c, c^{\prime}$ in $X_{\alpha}$. Then

$$
\begin{aligned}
\left|\underline{y}_{\alpha} \underline{y}_{\alpha}^{\prime}\right| & \geq\left|\underline{x}_{\alpha} \underline{x}_{\alpha}^{\prime}\right|+\cos 2 \epsilon\left(T_{\alpha}+T_{\alpha}^{\prime}\right) \\
& \geq\left(\left|x_{\alpha} x_{\alpha}^{\prime}\right|+T_{\alpha}+T_{\alpha}^{\prime}\right) \cos 2 \epsilon,
\end{aligned}
$$

while

$$
\begin{aligned}
\left|\bar{y}_{\alpha} \bar{y}_{\alpha}^{\prime}\right| & \leq\left|\bar{x}_{\alpha} \bar{x}_{\alpha}^{\prime}\right|+\left|\bar{c}_{\alpha}\right|+\left|\bar{c}_{\alpha}^{\prime}\right| \\
& \leq\left(\left|x_{\alpha} x_{\alpha}^{\prime}\right|+T_{\alpha}+T_{\alpha}^{\prime}\right) \sin 2 \epsilon .
\end{aligned}
$$

Thus

$$
\left|\bar{y}_{\alpha} \bar{y}_{\alpha}^{\prime}\right| /\left|\underline{y}_{\alpha} \underline{y}_{\alpha}^{\prime}\right| \leq \tan 2 \epsilon,
$$

i.e. the segment $y_{\alpha} y_{\alpha}^{\prime}$ forms the angle at most $2 \epsilon$ with $\mathbb{R}_{\alpha}$. Let $\beta \in w_{c}$ be the next letter after $\alpha$. Then $x_{\beta}=y_{\alpha}, x_{\beta}^{\prime}=y_{\alpha}^{\prime}$, and the angle between $\mathbb{R}_{\beta}$ and $\mathbb{R}_{\alpha}$ is uniformly separated from zero by $\omega>0$. Thus $x_{\beta} x_{\beta}^{\prime}$ forms the angle $\geq \omega / 2$ for $\epsilon<\omega / 4$ with the direction of $\mathbb{R}_{\beta}$, and we obtain a contradiction as above.

2.6.1. It follows that our decomposition

$$
\partial_{\infty} X=\mathcal{F} \cup \bigcup_{w \in \mathcal{W}} \mathcal{I}_{w}
$$

consists of connected (in the Tits metric) subsets, and each $\mathcal{I}_{w}$ is closed and nonempty.

2.7. Proposition. The set $\mathcal{F}$ is a connected component of $\partial_{T} X$.

Proof. It suffices to show that any geodesic segment $\gamma \subset \partial_{T} X$ with ends in $\mathcal{F}$ actually lies in $\mathcal{F}$.

Assume that it is not the case, and for any $\epsilon>0$ there is a point $z \in \gamma \cap \mathcal{I}$ with $\left|z z_{0}\right|<\epsilon$, where $z_{0} \in \mathcal{F}$ is the initial point of $\gamma$.

Let $w \in \mathcal{W}$ with $z \in \mathcal{I}_{w}$. The interval $\left(z_{0} z\right] \subset \gamma$ cannot lie in $\mathcal{I}_{w}$, since otherwise by 2.4.2 we have $z_{0} \in \mathcal{I}_{w}$, a contradiction. Furthermore, by closedness of $\mathcal{I}_{w}$ we can assume that $z^{\prime} \neq \mathcal{I}_{w}$ for any $z^{\prime} \in\left[z_{0} z\right)$. Then $\left(z_{0} z\right)$ necessarily contains a point $z^{\prime} \in \mathcal{F}$ (cp. the first argument in Lemma 2.8). It suffices to show that the segment $z_{0} z^{\prime}$ lies in $\mathcal{F}$.

By definition, the strings $w_{c_{0}}, w_{c^{\prime}}$ are finite for any $x \in X$, where the rays $c_{0} \in z_{0}$, $c^{\prime} \in z^{\prime}$ emanate from $x$. We can always choose $x \in X$ such that $w_{c_{0}}, w_{c^{\prime}}$ have at most one common letter. For a pair $\alpha, \alpha^{\prime}$ of consecutive letters of $w=w_{c_{0}} \cup w_{c^{\prime}}$ let $S_{\alpha \alpha^{\prime}} \subset \partial_{T} X$ be the boundary circle of the separating flat $X_{\alpha} \cap X_{\alpha^{\prime}}$. For three consecutive letters $\alpha_{0} \alpha_{1} \alpha_{2} \subset w$ the circles $S_{\alpha_{0} \alpha_{1}}, S_{\alpha_{1} \alpha_{2}}$ have only two common points, which are singular, and for the letter $\alpha_{3} \in w$ next after $\alpha_{2}$ the circles $S_{\alpha_{0} \alpha_{1}}, S_{\alpha_{2} \alpha_{3}}$ are disjoint. The singular points of $\partial_{T} X$ are pairwise separated by a 
distance $\geq \omega>\epsilon$, every noncontractible loop in $\partial_{T} X$ has length at least $2 \pi$, and $\operatorname{dim} \partial_{T} X=1$ by a result of B. Kleiner $\underline{\mathrm{Kl}}$.

All this implies that $w$ has at most three letters, and $z_{0} z^{\prime} \subset S_{\alpha_{0} \alpha_{1}} \cup S_{\alpha_{1} \alpha_{2}} \subset$ $\mathcal{F}$.

2.8. Lemma. If points $z, z^{\prime} \in \mathcal{I}$ are in the same connected component of $\partial_{T} X$, then $z, z^{\prime} \in \mathcal{I}_{w}$ for some $w \in \mathcal{W}$.

Proof. If the classes $f(z), f\left(z^{\prime}\right) \in \mathcal{W}$ are different, then any continuous curve $\gamma \subset \partial_{\infty} X$ between $z, z^{\prime}$ intersects the boundary $\partial_{\infty} E$ of at least one separating flat $E \subset X$, hence, $\gamma \cap \mathcal{F} \neq \emptyset$.

By the assumption, $z, z^{\prime}$ are connected by a geodesic $\gamma \subset \partial_{T} X$. Then $\gamma$ is continuous in $\partial_{\infty} X$. It follows that if $f(z) \neq f\left(z^{\prime}\right)$, then $\gamma$ intersects $\mathcal{F}$ and $\gamma \subset \mathcal{F}$. Thus $z, z^{\prime} \in \mathcal{F}$, a contradiction. Hence, $f(z)=f\left(z^{\prime}\right)$ and $z, z^{\prime} \in \mathcal{I}_{w}$ for some $w \in \mathcal{W}$.

From 2.6-2.8 we obtain

2.9. Corollary. The decomposition

$$
\partial_{T} X=\mathcal{F} \cup \bigcup_{w \in \mathcal{W}} \mathcal{I}_{w}
$$

coincides with the decomposition of $\partial_{T} X$ into connected components.

2.9.1. We shall call $\mathcal{F}$ the principal component of $\partial_{T} X$ and $\mathcal{I}_{w}, w \in \mathcal{W}$ nonprincipal components. By 2.4.1, 2.6 and 2.8, the map $f: \mathcal{I} \rightarrow \mathcal{W}$ induces a bijection between the set of nonprincipal components and $\mathcal{W}$. In the sequel, we identify $\mathcal{W}$ with the set of nonprincipal connected components of $\partial_{T} X$. Therefore, the last depends only on the fundamental group of $M$.

2.9.2. The principal component $\mathcal{F}$ of $\partial_{T} X$ is also independent of the metric $g$ on $M$ in the sense that for any other nonpositively curved metric $g^{\prime}$ on $M$ there is a canonical homeomorphism $\mathcal{F} \rightarrow \mathcal{F}^{\prime}$. This follows from the next two obvious facts.

(a) The vertices of the graph $\mathcal{F}$ are fixed points of the stabilizers $\mathrm{St}_{\alpha}$ of blocks $X_{\alpha} \subset X$.

(b) Any edge of $\partial_{T} X_{\alpha}$ has length $\pi$ and corresponds to a point of $\partial_{\infty} Y_{\alpha}, \alpha \in \mathcal{A}$. Since the metric on $Y_{\alpha}$ is hyperbolic, $\partial_{\infty} Y_{\alpha}$ is a quasi-isometric invariant of $Y_{\alpha}$.

Now we consider consequences of a rigid deformation of nonpositively curved metric on $M$. The next result generalizes an example from $[\mathrm{Bu}$.

2.10. Theorem. If for metrics $g_{0}, g_{1}$ of nonpositive curvature on a graphmanifold $M$ there is a continuous $\Gamma$-equivariant map $\partial_{\infty} X_{0} \rightarrow \partial_{\infty} X_{1}, \Gamma=\pi_{1}(M)$, then the principal components $\mathcal{F}_{0}, \mathcal{F}_{1}$ of the Tits boundaries $\partial_{T} X_{0}, \partial_{T} X_{1}$ are canonically isometric.

Proof. If $\mathcal{F}_{0}, \mathcal{F}_{1}$ are not isometric, then for some adjacent blocks $X_{\alpha}, X_{\alpha^{\prime}} \subset X$ we have $\angle_{0}\left(\alpha, \alpha^{\prime}\right) \neq \angle_{1}\left(\alpha, \alpha^{\prime}\right)$ for the Tits distances between corresponding singular points $\alpha, \alpha^{\prime} \in \partial_{T} X$. The boundary circle $S$ of the separating flat $E=X_{\alpha} \cap X_{\alpha^{\prime}}$ is the union of two edges $S=e_{0} \cup e_{1}$ of length $\pi$ between $\alpha$ and $-\alpha$ (in any metric $\left.g_{0}, g_{1}\right)$, and $\alpha^{\prime},-\alpha^{\prime} \in S$. We can assume that $\alpha^{\prime}$ is an interior point of $e_{0}$.

We take $\beta \in e_{1}$ with $\angle_{0}(\beta, \alpha)=\angle_{0}\left(\alpha, \alpha^{\prime}\right)$ and consider $\gamma \in \Gamma$, which represents a nonperipheral element of $\pi_{1}\left(F_{v}\right) \subset \Gamma$, where $M_{v}=F_{v} \times S^{1}$ is the maximal block 
covered by $X_{\alpha}$. Then $\gamma(\alpha)=\alpha, \gamma(-\alpha)=-\alpha$, and $\gamma^{n}\left(e_{0}\right), \gamma^{n}\left(e_{1}\right)$ converge in $\partial_{\infty} X$ to the same edge $e \subset \partial_{T} X$ between $\alpha$ and $-\alpha$ (the edge $e$ is defined by one of the ends $z \in \partial_{\infty} Y_{\alpha}$ of an axis of $\gamma$ in $\left.Y_{\alpha}\right)$. Furthermore, the sequences $\gamma^{n}\left(\alpha^{\prime}\right), \gamma^{n}(\beta)$ converge in $\partial_{\infty} X_{0}$ to the same point of $e$ by the choice of $\beta$. However, for the metric $g_{1}$ we have $L_{1}(\beta, \alpha) \neq \angle_{1}\left(\alpha, \alpha^{\prime}\right)$, and the sequences $\gamma^{n}\left(\alpha^{\prime}\right), \gamma^{n}(\beta)$ converge in $\partial_{\infty} X_{1}$ to different points of $e$ (the point $\beta \in e_{1}$ being fixed can be approximated by ends of axis of elements from the stabilizer $\mathrm{St}_{E} \subset \Gamma$ of $E$, since $\mathrm{St}_{E} \simeq \mathbb{Z}^{2}$, hence it is well defined and for the metric $\left.g_{1}\right)$.

Therefore, there is no continuous $\Gamma$-equivariant map $\partial_{\infty} X_{0} \rightarrow \partial_{\infty} X_{1}$.

We have the same sort of effects for a soft deformation, if the last results in a degeneration of a nonprincipal component.

2.11. Proposition. Assume that for nonpositively curved metrics $g_{0}, g_{1}$ on a graphmanifold $M$ there is a $w \in \mathcal{W}$ such that the connected component $\mathcal{I}_{w}$ is degenerate with respect to $g_{0}$ and distinct from a point w.r.t. $g_{1}$. Then there exists no continuous $\Gamma$-equivariant map $\partial_{\infty} X_{0} \rightarrow \partial_{\infty} X_{1}$ for $\Gamma=\pi_{1}(M)$.

Proof. Fix some string $w_{c} \in w$ and consider the sequence $S_{\alpha}=\partial_{\infty} E_{\alpha}, \alpha \in w_{c}$ of circles on $\partial_{\infty} X_{0}, \partial_{\infty} X_{1}$, where $E_{\alpha}$ is the initial w.r.t. $w_{c}$ separating flat of the block $X_{\alpha}$. If a letter $\beta \in w_{c}$ follows $\alpha$ and is not the next one, then $S_{\beta} \subset \mathcal{D}_{\alpha}$, where $\mathcal{D}_{\alpha} \subset \partial_{\infty} X$ is an open subset homeomorphic to the open disc and bounded by $S_{\alpha}$ (we suppress the subsrcipts 0,1 ).

The collection $\left\{\mathcal{D}_{\alpha}\right\}_{\alpha \in w_{c}}$ is a basis of neighborhoods in $\partial_{\infty} X$ of the component $\mathcal{I}_{w}$, in particular, $\bigcap_{\alpha \in w_{c}} \mathcal{D}_{\alpha}=\mathcal{I}_{w}$.

Therefore, for $g_{0}$ the sets $S_{\alpha}$ converge in $\partial_{\infty} X_{0}$ to the point $\mathcal{I}_{w}$ as $\alpha \rightarrow \infty$. On the other hand, since $\mathcal{I}_{w}$ is nondegenerate for $g_{1}$, we can find $x_{\alpha}, y_{\alpha} \in S_{\alpha}$ such that

$$
\lim _{\alpha \rightarrow \infty} x_{\alpha} \neq \lim _{\alpha \rightarrow \infty} y_{\alpha}
$$

in $\partial_{\infty} X_{1}$, while $\lim _{\alpha \rightarrow \infty} x_{\alpha}=\lim _{\alpha \rightarrow \infty} y_{\alpha}=\mathcal{I}_{w}$ in $\partial_{\infty} X_{0}$. One can always assume that the points $x_{\alpha}, y_{\alpha}$ are rational, i.e. correspond to some cyclic subgroups in $\Gamma$. Thus there is no continuous $\Gamma$-equivariant map $\partial_{\infty} X_{0} \rightarrow \partial_{\infty} X_{1}$.

2.12. Proposition. Each nonprincipal component $\mathcal{I}_{w}, w \in \mathcal{W}$ is a segment in $\partial_{\infty} X$ of length $<\pi$ and may be degenerate.

For the proof we consider the following invariant of a point $z \in \mathcal{I}_{w}$. For a ray $c \in z$ and $\alpha \in w_{c}$ let $\eta_{c}(\alpha)$ be the angle between $c$ and the boundary component of $X_{\alpha}$ in the entering point $x_{\alpha}$ of $c$ into $X_{\alpha}$. We define

$$
\eta_{c}=\limsup _{\alpha \rightarrow \infty} \eta_{c}(\alpha)
$$

2.12.1. Lemma. The number $\eta_{c}$ is independent on the ray $c \in z$, i.e. $\eta_{c}=\eta_{z}$ for all $c \in z$.

Proof. Let $c, c^{\prime} \in z$ be some rays. One can assume that $w_{c}=w_{c^{\prime}}$. It easily follows from properties of asymptotic rays that for sufficiently large $\alpha \in w_{c}$ the tangent vectors $\dot{c}_{\alpha}, \dot{c}_{\alpha}^{\prime}$ to $c, c^{\prime}$ at the entering points $x_{\alpha}, x_{\alpha}^{\prime}$ in $X_{\alpha}$ are almost parallel, i.e. by applying the parallel translation along $x_{\alpha} x_{\alpha}^{\prime}$ they differ by a vector of size $\epsilon_{\alpha}$, where $\epsilon_{\alpha} \rightarrow 0$ as $\alpha \rightarrow \infty$. It gives $\eta_{c}=\eta_{c^{\prime}}$.

The following lemma is the key one for the proof of Proposition 2.12. 
2.12.2. Lemma. If $\eta_{z} \neq 0$ for some point $z \in \mathcal{I}_{w}$, then the component $\mathcal{I}_{w}$ is degenerate, $\mathcal{I}_{w}=\{z\}$.

Proof. Assume that it is not the case and the component $\mathcal{I}_{w}$ is nondegenerate. Then for each sufficiently small $\epsilon>0$ there is a point $z^{\prime} \in \mathcal{I}_{w}, z^{\prime} \neq z$ with $\angle\left(z, z^{\prime}\right)=\epsilon$. We take $\epsilon$ on a scale of sizes arbitrarily smaller than $\eta=\eta_{z}, \epsilon \prec \eta$.

For any $\sigma>0, \sigma \prec \epsilon$ there is a point $x \in X$ such that $\angle_{x}\left(z, z^{\prime}\right) \geq \angle\left(z, z^{\prime}\right)-\sigma$. Let $c \in z, c^{\prime} \in z^{\prime}$ be the rays with the common vertex $x$. Then for any $t, t^{\prime}>0$ the triangle $x c(t) c^{\prime}\left(t^{\prime}\right)$ bounds in $X$ a ruled $\sigma$-almost flat surface, i.e. whose absolute integral curvature is $\leq \sigma$. In particular, the vectors $\dot{c}(t), \dot{c}_{\|}^{\prime}\left(t^{\prime}\right) \in T_{c(t)} X$ form the angle $\sigma$-close to $\epsilon$ and lie $\sigma$-almost in the one 2-subspace with the direction of the segment $c(t) c^{\prime}\left(t^{\prime}\right)$. Here $\dot{c}_{\|}^{\prime}\left(t^{\prime}\right)$ is the parallel translate of $\dot{c}^{\prime}\left(t^{\prime}\right)$ along $c^{\prime}\left(t^{\prime}\right) c(t)$.

By the assumption, there is an infinite sequence of letters $\alpha \in w_{c}$, for which the direction of $c$ at the entering point $x_{\alpha}$ into $X_{\alpha}$ forms the angle $\eta_{c}(\alpha) \geq \eta$ with the corresponding separating flat $E_{\alpha}$. We denote $x_{\alpha}^{\prime}=c^{\prime} \cap E_{\alpha}$.

The segment $x_{\alpha} x_{\alpha}^{\prime}$ forms the angle $\phi_{\alpha} \geq \omega / 2$ with one of the $\mathbb{R}$-factors $\mathbb{R}_{\alpha}$ of $X_{\alpha}$ or $\mathbb{R}_{\beta}$ of the preceding block $X_{\beta}$, where $\omega$ is the minimal angle between singular directions of adjacent blocks in $X$.

First, consider the case when it is the factor $\mathbb{R}_{\alpha}$. Let $\bar{x}_{\alpha} \bar{x}_{\alpha}^{\prime}$ be the projection of $x_{\alpha} x_{\alpha}^{\prime}$ on the factor $Y_{\alpha}$ from the decomposition $X_{\alpha}=Y_{\alpha} \times \mathbb{R}$. Then $\left|\bar{x}_{\alpha} \bar{x}_{\alpha}^{\prime}\right| \rightarrow \infty$ as $\alpha \rightarrow \infty$. Furthermore, the segments $\bar{c}_{\alpha}, \bar{c}_{\alpha}^{\prime}$ form the angles $\psi_{\alpha}, \psi_{\alpha}^{\prime}$ with $\bar{x}_{\alpha} \bar{x}_{\alpha}^{\prime}$, which are $(\eta-\epsilon)$-separated from zero and $\pi$, where $\bar{c}_{\alpha}, \bar{c}_{\alpha}^{\prime}$ are the projections of the segments $c_{\alpha}=c \cap X_{\alpha}, c_{\alpha}^{\prime}=c^{\prime} \cap X_{\alpha}$ on $Y_{\alpha}$.

Using the conditions $\eta_{c}(\alpha) \geq \eta, \phi_{\alpha} \geq \omega / 2$ and the above remark about the triangles $x c(t) c^{\prime}\left(t^{\prime}\right)$, it is not difficult to see that $\psi_{\alpha}+\psi_{\alpha}^{\prime} \geq \pi+\lambda \cdot \epsilon-\sigma$, where the coefficient $\lambda>0$ depends only on $\eta$ and $\omega$. Thus $\psi_{\alpha}+\psi_{\alpha}^{\prime}>\pi$. In that case the periodicity argument immediately gives a contradiction.

Assume now that $x_{\alpha} x_{\alpha}^{\prime}$ forms the angle $\phi_{\alpha} \geq \omega / 2$ with the $\mathbb{R}$-factor $\mathbb{R}_{\beta}$ of the preceding block $X_{\beta}$. Then repeating the arguments above and replacing $\alpha$ by $\beta$, we obtain that the angles $\psi_{\beta}, \psi_{\beta}^{\prime}$ are $(\eta-\epsilon)$-separated from 0 and $\pi$. This time we only have $\pi \geq \psi_{\beta}+\psi_{\beta}^{\prime} \geq \pi-\lambda \cdot \epsilon-\sigma$, where the coefficient $\lambda<\infty$ depends only on $\eta$ and $\omega$. Choosing $\epsilon$ sufficiently small (depending only on $\eta$ and $\omega$ ), letting $\alpha \rightarrow \infty$ and applying the periodicity argument, we find in $Y_{\beta} \sigma$-almost flat triangles with arbitrary large size of the inscribed disc. This is, obviously, impossible.

Proof of Proposition 2.12. Assume that a component $\mathcal{I}_{w} \subset \partial_{T} X$ is nondegenerate. Then by Lemma 2.12.2, $\eta_{z}=0$ for all $z \in \mathcal{I}_{w}$. Now let $z, z^{\prime}, z^{\prime \prime} \in \mathcal{I}_{w}$ be pairwise different sufficiently close points, and let $\epsilon>0$ be the minimal distance between them. For any $\sigma>0, \sigma \prec \epsilon$ we can find $x \in X$ such that for the rays $c \in z, c^{\prime} \in z^{\prime}$, $c^{\prime \prime} \in z^{\prime \prime}$ emanating from $x$ the measure of nonflatness of any sectors $c c^{\prime}, c c^{\prime \prime}, c^{\prime} c^{\prime \prime}$ is less than $\sigma$. Moving forward along $c$ and using the condition $\eta_{z}=\eta_{z^{\prime}}=\eta_{z^{\prime \prime}}=0$, we find that for sufficiently large $\alpha \in w_{c}$ the direction of $c$ at the entering point $x_{\alpha}$ into $X_{\alpha}$ is $\sigma$-close to the separating flat $E_{\alpha}$, and this is also true for the rays $x_{\alpha} z^{\prime}$, $x_{\alpha} z^{\prime \prime}$. It immediately follows that one of the points $z, z^{\prime}, z^{\prime \prime} \in \mathcal{I}_{w}$ lies between two others.

2.13. Action of the fundamental group $\Gamma$ on the set of connected components of $\partial_{T} X$.

The principal component $\mathcal{F}$, obviously, is invariant for any $\gamma \in \Gamma$. No one component $\mathcal{I}_{w}$ is invariant for whole $\Gamma$, however there always exist nonprincipal 
components $\mathcal{I}_{w}$, which are invariant for some nontrivial $\gamma \in \Gamma$. If it is the case, then $\gamma$ is a rank 1 isometry in the sense of $\left[\mathrm{BB}\right.$, and $\mathcal{I}_{w}$ is degenerate for any nonpositively curved metric on $M$. Such $\gamma$ represents a closed geodesic in $M$, which is not contained in one block $M_{v}$ of $M$.

A connected component of $\partial_{T} X$ is said to be standard, if it is invariant at least for one nontrivial $\gamma \in \Gamma$. The other components are called nonstandard.

\section{The LENGth SPECTRUM OF NONStANDARD COMPONENTS}

We use notation $L(w)$ for the length of a nonstandard connected component $\mathcal{I}_{w} \subset \partial_{T} X, w \in \mathcal{W}$.

We start with the proof of Theorem 0.1. For that we need the following simple lemma (its proof we leave to the reader).

3.1. Lemma. Assume that unit vectors $v, v^{\prime}, \alpha, \beta \in \mathbb{R}^{2}$ satisfy the conditions $\angle\left(v, v^{\prime}\right)>\omega \geq \pi / 2$ and $\pi / 2 \leq \angle(\alpha, \beta) \leq \omega$.

If the projections $\bar{v}_{\alpha}, \bar{v}_{\alpha}^{\prime}$ of $v, v^{\prime}$ on the line $\mathbb{R} \cdot \alpha$ are codirected, then the projections $\bar{v}_{\beta}, \bar{v}_{\beta}^{\prime}$ on $\mathbb{R} \cdot \beta$ are nonzero and opposite directed.

Proof of Theorem 0.1. Assume to the contrary that for some $w \in \mathcal{W}$ there exist $z$, $z^{\prime} \in \mathcal{I}_{w}$ with $\angle\left(z, z^{\prime}\right)>\omega$. We put $\epsilon=\angle\left(z, z^{\prime}\right)-\omega$. Then $\epsilon<\pi-\omega$.

For any $\sigma>0$ there is $x \in X$ with $\angle_{x}\left(z, z^{\prime}\right) \geq \omega+\epsilon-\sigma$. Then for the rays $c \in z, c^{\prime} \in z^{\prime}$ emanating from $x$ and any $t, t^{\prime}>0$ the triangle $x c(t) c^{\prime}\left(t^{\prime}\right)$ bounds in $X$ a ruled $\sigma$-almost flat surface with total curvature $\leq \sigma$. Furthermore, by Lemma 2.12.2, one can assume that the rays $c, c^{\prime}$ form angles $\leq \sigma$ with the separating flat $E_{\alpha}$ at the entering points $x_{\alpha}=c \cap E_{\alpha}, x_{\alpha}^{\prime}=c^{\prime} \cap E_{\alpha}$ into the block $X_{\alpha}$ for all $\alpha \in w_{c}$.

Moving forward along the string $w_{c}$ one can make $\sigma$ arbitrary small comparing to $\epsilon$; thus we assume that $\sigma \prec \epsilon$. It means, in particular, that we denote by the same symbol $\sigma$ the constants of the scale of $\sigma$.

Let $\bar{c}_{\alpha}, \bar{c}_{\alpha}^{\prime}$ be the projections of the segments $c_{\alpha}=c \cap X_{\alpha}, c_{\alpha}^{\prime}=c^{\prime} \cap X_{\alpha}$ on the factor $Y_{\alpha}$ from the decomposition $X_{\alpha}=Y_{\alpha} \times \mathbb{R}$. We denote by $\mathbb{R}_{\alpha}$ the $\mathbb{R}$-factor of that decomposition. In the sequel, we use the affine structure of the flat $E_{\alpha}$. Speaking about the angle between a vector $v \in E_{\alpha}$ and $\mathbb{R}_{\alpha}$ we mean the angle between $v$ and its projection on $\mathbb{R}_{\alpha}$ (such an angle is always $\leq \pi / 2$ ).

Let $v_{\alpha}, v_{\alpha}^{\prime}$ be the unit vectors in $E_{\alpha}$, which are the projections of the directions of the rays $c, c^{\prime}$ at $x_{\alpha}, x_{\alpha}^{\prime}$. We say that there is a sufficient separation (from the $\mathbb{R}$-factor) for $\alpha \in w_{c}$, if $\angle\left(v_{\alpha}, \mathbb{R}_{\alpha}\right), \angle\left(v_{\alpha}^{\prime}, \mathbb{R}_{\alpha}\right) \geq \epsilon / 4$.

Let $\bar{v}_{\alpha}, \bar{v}_{\alpha}^{\prime}$ be the projections of $v_{\alpha}, v_{\alpha}^{\prime}$ on the factor $Y_{\alpha}$. We say that we have opposite projections for $\alpha \in w_{c}$, if the vectors $\bar{v}_{\alpha}, \bar{v}_{\alpha}^{\prime}$ are nonzero and opposite directed; otherwise we have codirected projections for $\alpha \in w_{c}$.

Step 1. Assume that for $\alpha \in w_{c}$ there is a sufficient separation from $\mathbb{R}_{\alpha}$ and opposite projections. We shall show that this leads to a contradiction.

The vectors $v_{\alpha}, v_{\alpha}^{\prime}$ form the angles $\phi_{\alpha}, \phi_{\alpha}^{\prime}$ with the segment $x_{\alpha} x_{\alpha}^{\prime}$, whose sum differs from $\pi+\omega+\epsilon$ at most by $\sigma$. The angle $\angle\left(v_{\alpha}, v_{\alpha}^{\prime}\right)$ also differs from $\omega+\epsilon$ at most by $\sigma$. It easily follows from this that

3.1.1. If $\phi_{\alpha}, \phi_{\alpha}^{\prime} \leq \pi-\epsilon / 8$, then the vectors $v_{\alpha}, v_{\alpha}^{\prime}$ are directed in the same halfplane in $E_{\alpha}$ with respect to the line of the segment $x_{\alpha} x_{\alpha}^{\prime}$.

Now, if $x_{\alpha} x_{\alpha}^{\prime}$ forms the angle $<\epsilon / 8$ with $\mathbb{R}_{\alpha}$, then $\phi_{\alpha}, \phi_{\alpha}^{\prime} \leq \pi-(\epsilon / 4-\epsilon / 8)=$ $\pi-\epsilon / 8$. Thus by 3.1.1, the vectors $v_{\alpha}, v_{\alpha}^{\prime}$ are on one side with respect to $x_{\alpha} x_{\alpha}^{\prime}$. 
Using the sufficient separation from $\mathbb{R}_{\alpha}$, we obtain that the projections $\bar{v}_{\alpha}, \bar{v}_{\alpha}^{\prime}$ are codirected, which contradicts our assumption.

Hence, $x_{\alpha} x_{\alpha}^{\prime}$ forms the angle $\geq \epsilon / 8$ with $\mathbb{R}_{\alpha}$. Let $\bar{x}_{\alpha}, \bar{x}_{\alpha}^{\prime}$ be the projections of $x_{\alpha}, x_{\alpha}^{\prime}$ on $Y_{\alpha}$. Since $\left|x_{\alpha} x_{\alpha}^{\prime}\right| \rightarrow \infty$ as $\alpha \rightarrow \infty$, we can assume that $\left|\bar{x}_{\alpha} \bar{x}_{\alpha}^{\prime}\right| \geq \rho_{1}$ (see Lemma 2.2.5). The segments $\bar{c}_{\alpha}, \bar{c}_{\alpha}^{\prime} \subset Y_{\alpha}$ form the angles $\leq \sigma$ with the boundary component of $Y_{\alpha}$ corresponding to $E_{\alpha}$. Using again 3.1.1, we obtain that $\bar{c}_{\alpha}, \bar{c}_{\alpha}^{\prime}$ form the angles $\geq \pi-\sigma$ with the segment $\bar{x}_{\alpha} \bar{x}_{\alpha}^{\prime}$. Then the periodicity argument immediately gives a contradiction.

Step 2. Assume that there is no sufficient separation from $\mathbb{R}_{\alpha}$ for $\alpha \in w_{c}$. Let $\beta \in w_{c}$ be the next letter after $\alpha$. We shall show that for $\beta$ we have a sufficient separation from $\mathbb{R}_{\beta}$ and opposite projections.

Without loss of generality, we assume that $\angle\left(v_{\alpha}, \mathbb{R}_{\alpha}\right)<\epsilon / 4$. Then, obviously, $\angle\left(v_{\beta}, \mathbb{R}_{\alpha}\right) \leq \epsilon / 4+\sigma$ (here the vector $v_{\beta}$ and the factor $\mathbb{R}_{\alpha}$ are considered in the flat $\left.E_{\beta}\right)$. Thus $\angle\left(v_{\beta}, \mathbb{R}_{\beta}\right) \geq \pi-\omega-\epsilon / 4-\sigma \geq \epsilon / 4$. Furthemore, $\angle\left(v_{\beta}^{\prime}, \mathbb{R}_{\beta}\right) \geq$ $\angle\left(v_{\beta}, v_{\beta}^{\prime}\right)-\angle\left(v_{\beta}, \mathbb{R}_{\alpha}\right)-\omega \geq \epsilon / 4$. Moreover, the vectors $v_{\beta}, v_{\beta}^{\prime}$ are directed into different half-planes in $E_{\beta}$ with respect to $\mathbb{R}_{\beta}$, i.e. $\bar{v}_{\beta}, \bar{v}_{\beta}^{\prime}$ are opposite directed.

Therefore, for $\beta$ we have a sufficient separation from $\mathbb{R}_{\beta}$ and opposite projections. By Step 1, this leads to a contradiction.

Step 3. Hence, for $\alpha \in w_{c}$ we have a sufficient separation from $\mathbb{R}_{\alpha}$ and codirected projections. Let $\beta \in w_{c}$ be the next letter after $\alpha$. We shall show that for $\beta$ we have opposite projections.

The angles $\angle\left(v_{\alpha}, \mathbb{R}_{\alpha}\right), \angle\left(v_{\beta}, \mathbb{R}_{\alpha}\right)$ differ at most by $\sigma$, and the same is true for the pairs of angles $\angle\left(v_{\alpha}^{\prime}, \mathbb{R}_{\alpha}\right), \angle\left(v_{\beta}^{\prime}, \mathbb{R}_{\alpha}\right)$ and $\angle\left(v_{\alpha}, v_{\alpha}^{\prime}\right), \angle\left(v_{\beta}, v_{\beta}^{\prime}\right)$. Since for $\alpha$ we have codirected projections, it means that

$$
\angle\left(v_{\alpha}, \mathbb{R}_{\alpha}\right)+\angle\left(v_{\alpha}, v_{\alpha}^{\prime}\right)+\angle\left(v_{\alpha}^{\prime}, \mathbb{R}_{\alpha}\right)=\pi .
$$

Assume that the projections of $v_{\beta}, v_{\beta}^{\prime}$ on $Y_{\alpha}$ (in the flat $E_{\beta}$ ) are opposite directed. Then, without loss of generality, we have

$$
\angle\left(v_{\beta}, \mathbb{R}_{\alpha}\right)+\angle\left(v_{\beta}, v_{\beta}^{\prime}\right)-\angle\left(v_{\beta}^{\prime}, \mathbb{R}_{\alpha}\right)=\pi .
$$

Thus

$$
\begin{aligned}
\angle\left(v_{\beta}, \mathbb{R}_{\alpha}\right)-\angle\left(v_{\alpha}, \mathbb{R}_{\alpha}\right) & =\angle\left(v_{\alpha}^{\prime}, \mathbb{R}_{\alpha}\right)+\angle\left(v_{\beta}^{\prime}, \mathbb{R}_{\alpha}\right)+\angle\left(v_{\alpha}, v_{\alpha}^{\prime}\right)-\angle\left(v_{\beta}, v_{\beta}^{\prime}\right) \\
& \geq \epsilon / 2-\sigma,
\end{aligned}
$$

which contradicts the estimate $\angle\left(v_{\beta}, \mathbb{R}_{\alpha}\right)-\angle\left(v_{\alpha}, \mathbb{R}_{\alpha}\right) \leq \sigma$.

Therefore, the projections of $v_{\beta}, v_{\beta}^{\prime}$ on $Y_{\alpha}$ are codirected. It follows from Lemma 3.1 that for $\beta$ we have opposite projections. By Step 2, we also have a sufficient separation for $\beta$. By Step 1, this leads to a contradiction, which completes the proof of Theorem 0.1.

\section{Nonstandard components of maximal length}

We show here that the estimate of Theorem 0.1 is optimal, in particular, that nondegenerate nonstandard components do exist.

3.2. A nonstandard component $\mathcal{I}_{w}$ is said to be primitive, if the separating flats $E_{\alpha}$ for all $\alpha \in w_{c}$ belong to the same orbit of the action of $\Gamma=\pi_{1}(M)$ for some $w_{c} \in w$.

In other words, it means that any geodesic ray $c:[0, \infty) \rightarrow X, c(\infty) \in \mathcal{I}_{w}$ descends to a geodesic $\bar{c}$ in $M$, which starting from some moment intersects only 
one gluing torus $T_{u} \subset M$ and hence $\bar{c}$ lives only in two adjacent maximal blocks $M_{v}, M_{v^{\prime}}$ of $M$ skipping from one to another. We use the notation $\mathcal{W}_{u}$ for the set of the nonprincipal connected components of $\partial_{T} X$ associated with $T_{u}$.

3.2.1. Remark. We have actually proved that for any primitive component $w \in \mathcal{W}_{u}$ its length satisfies $L(w) \leq \omega_{u}$, where $\omega_{u} \in[\pi / 2, \pi)$ is the angle between $S^{1}$-factors on $T_{u}$.

3.3. Here we prove Theorem 0.2 . To each singular point $\alpha \in \mathcal{F}$ it corresponds exactly one block $X_{\alpha}$ with singular direction $\alpha$. Any singular point $\beta \in \mathcal{F}$ is connected with $\alpha$ by a minimizer $\alpha \beta \subset \mathcal{F}$. If some minimizer $\alpha \beta$ contains no other singular point, then the blocks $X_{\alpha}, X_{\beta}$ are adjacent, $\alpha \beta$ lies in the boundary at infinity $S_{\alpha \beta}$ of the separating flat $E_{\alpha \beta}=X_{\alpha} \cap X_{\beta}$ and $\angle(\alpha, \beta) \leq \omega(g)$. In that case the points $\alpha, \beta$ are called neighboring. We say that a pair of neighboring singular points $(\alpha, \beta)$ belongs to the class $\mathcal{W}_{u}$, if the separating flat $E_{\alpha \beta}$ covers the torus $T_{u}$ and $\angle(\alpha, \beta)=\omega_{u}$.

3.3.1. Lemma. Let $(\alpha, \beta)$ be a pair of neighboring points of a class $\mathcal{W}_{u}$. Then there exists a sequence of singular points $\beta_{n} \in \mathcal{F}$ such that

(i) $\beta_{n} \rightarrow \alpha$ in the standard topology;

(ii) the pairs $\left(\beta_{n}, \alpha\right)$ belong to the class $\mathcal{W}_{u}$.

Proof. Applying to $X_{\alpha}$ the isometries from $\mathrm{St}_{\beta} \backslash \mathrm{St}_{\alpha}$, where $\mathrm{St}_{\beta} \subset \Gamma$ is the stabilizer of the block $X_{\beta}$, we obtain infinitely many blocks $X_{\beta^{\prime}}$ adjacent to $X_{\beta}$, for which $\left(\beta, \beta^{\prime}\right)$ belong to the class $\mathcal{W}_{u}$. Choosing an isometry $\gamma \in \mathrm{St}_{\alpha} \cap \mathrm{St}_{\beta}$, whose axis $l_{\gamma}$ has the positive direction $l_{\gamma}(\infty)=\alpha$, and applying $\gamma^{n}, n \geq 1$ to $\beta^{\prime}$ we obtain a required sequence $\beta_{n}$.

3.3.2. Corollary. Let $(\alpha, \beta)$ be a pair of singular points of a class $\mathcal{W}_{u}$. Then for any neighborhood $U$ of $\alpha$ in $\partial_{\infty} X$ there exists a singular point $\alpha^{\prime} \in U \cap \mathcal{F}$ such that $\left(\beta, \alpha^{\prime}\right)$ belongs to the class $\mathcal{W}_{u}$ and $X_{\alpha} \neq X_{\alpha^{\prime}}$.

3.4. Proof of Theorem 0.2. We fix an $\epsilon>0$ and a sequence $\epsilon_{i}$ with $\sum_{i>0} \epsilon_{i} \leq \epsilon$. The required component $\mathcal{I}_{w} \subset \partial_{T} X$ will be obtained by the following inductive construction of a sequence of letters $\alpha_{0}, \beta_{0}, \alpha_{1}, \beta_{1} \ldots \in \mathcal{A}$ with $w=\alpha_{0} \beta_{0} \alpha_{1} \beta_{1} \ldots$

Step 0. Take a pair $\left(\alpha_{0}, \beta_{0}\right)$ of the class $\mathcal{W}_{u}$ and $x_{0} \in X$ such that the block $X_{\alpha_{0}}$ separates $x_{0}$ with $X_{\beta_{0}}$. Notation: $\alpha_{0}<\beta_{0}$.

We can identify any $z \in \partial_{\infty} X$ with the ray $c \in z$ emanating from $x_{0}$. In particular, $\alpha_{0}, \beta_{0}:[0, \infty) \rightarrow X$ are geodesic rays with $\alpha_{0}(0)=\beta_{0}(0)=x_{0}$ and $\alpha_{0}(\infty)=\alpha_{0}, \beta_{0}(\infty)=\beta_{0}$.

One can find $t_{0}>0$ such that for the comparison triangle $\bar{x}_{0} \bar{\alpha}_{0}\left(t_{0}\right) \bar{\beta}_{0}\left(t_{0}\right) \subset \mathbb{R}^{2}$ we have $\angle \bar{\alpha}_{0}\left(t_{0}\right) \bar{x}_{0} \bar{\beta}_{0}\left(t_{0}\right) \geq \omega_{u}-\epsilon_{0}$.

Moving forward along the rays $\alpha_{0}, \beta_{0}$ if necessary, we additionally assume that $\alpha_{0}\left(t_{0}\right)$ belongs to the block, preceding $X_{\alpha_{0}}$ (with respect to $x_{0}$ ), and $\beta_{0}\left(t_{0}\right) \in X_{\alpha_{0}}$.

Step 1. By Corollary 3.3.2, there exists a singular point $\alpha_{1} \in \mathcal{F}$ such that $\beta_{0}<\alpha_{1},\left(\beta_{0}, \alpha_{1}\right)$ belongs to the class $\mathcal{W}_{u}$ and $\left|\alpha_{0}\left(t_{0}\right) \alpha_{1}\left(t_{0}\right)\right| \leq \epsilon_{0}$.

Having chosen this point we can find $t_{1}>t_{0}$ such that for the comparison triangle $\bar{x}_{0} \bar{\alpha}_{1}\left(t_{1}\right) \bar{\beta}_{0}\left(t_{1}\right)$ we have $\angle \bar{\alpha}_{1}\left(t_{1}\right) \bar{x}_{0} \bar{\beta}_{0}\left(t_{1}\right) \geq \omega_{u}-\epsilon_{1}$. Again, one can additionally assume that $\alpha_{1}\left(t_{1}\right) \in X_{\beta_{0}}$ and $\beta_{0}\left(t_{1}\right) \in X_{\alpha_{0}}$.

Repeating this argument, we can find a singular point $\beta_{1} \in \mathcal{F}$ such that $\beta_{1}>\alpha_{1}$, the pair $\left(\alpha_{1}, \beta_{1}\right)$ belongs to the class $\mathcal{W}_{u}$ and $\left|\beta_{0}\left(t_{1}\right) \beta_{1}\left(t_{1}\right)\right| \leq \epsilon_{1}$. Then we find $t_{2}>t_{1}$ with $\angle \bar{\alpha}_{1}\left(t_{2}\right) \bar{x}_{0} \bar{\beta}_{1}\left(t_{2}\right) \geq \omega_{u}-\epsilon_{2}, \alpha_{1}\left(t_{2}\right) \in X_{\beta_{0}}, \beta_{1}\left(t_{2}\right) \in X_{\alpha_{1}}$. 
Step 2. As in Step 1, we find a singular point $\alpha_{i}>\beta_{i-1}$, for which the pair $\left(\beta_{i-1}, \alpha_{i}\right)$ belongs to the class $\mathcal{W}_{u}$, such that

$$
\left|\alpha_{i}\left(t_{2(i-1)}\right) \alpha_{i-1}\left(t_{2(i-1)}\right)\right| \leq \epsilon_{2(i-1)} .
$$

Moving forward along the rays $\alpha_{i}, \beta_{i-1}$, we find $t_{2 i-1}>t_{2(i-1)}$ such that

$$
\begin{gathered}
\angle \bar{\alpha}_{i}\left(t_{2 i-1}\right) \bar{x}_{0} \bar{\beta}_{i-1}\left(t_{2 i-1}\right) \geq \omega_{u}-\epsilon_{2 i-1}, \\
\alpha_{i}\left(t_{2 i-1}\right) \in X_{\beta_{i-1}}, \beta_{i-1}\left(t_{2 i-1}\right) \in X_{\alpha_{i-1}} .
\end{gathered}
$$

Likewise, we find $\beta_{i}>\alpha_{i}$, for which $\left(\alpha_{i}, \beta_{i}\right)$ belongs to the class $\mathcal{W}_{u}$, such that

$$
\left|\beta_{i}\left(t_{2 i-1}\right) \beta_{i-1}\left(t_{2 i-1}\right)\right| \leq \epsilon_{2 i-1} .
$$

Then we find $t_{2 i}>t_{2 i-1}$ with

$$
\begin{gathered}
\angle \bar{\alpha}_{i}\left(t_{2 i}\right) \bar{x}_{0} \bar{\beta}_{i}\left(t_{2 i}\right) \geq \omega_{u}-\epsilon_{2 i}, \\
\alpha_{i}\left(t_{2 i}\right) \in X_{\beta_{i-1}}, \beta_{i}\left(t_{2 i}\right) \in X_{\alpha_{i}} .
\end{gathered}
$$

This procedure generates an infinite string $w \in W$, which is primitive by the construction and its class belongs to $\mathcal{W}_{u}$. It follows from (3.4.1) and (3.4.4) that

$$
\left|\alpha_{i}\left(t_{j}\right) \alpha_{i-1}\left(t_{j}\right)\right| \leq \epsilon_{2(i-1)} \quad \text { and } \quad\left|\beta_{i}\left(t_{j}\right) \beta_{i-1}\left(t_{j}\right)\right| \leq \epsilon_{2 i-1}
$$

for all $0 \leq j \leq 2(i-1)$, which implies by the choice of $\left\{\epsilon_{i}\right\}$ that $\left\{\alpha_{i}\right\},\left\{\beta_{i}\right\}$ are Cauchy sequences of rays in $X$. For the limit rays $\alpha=\lim \alpha_{i}$ and $\beta=\lim \beta_{i}$ we have

$$
w_{\alpha}=w_{\beta}=w,
$$

i.e. $\alpha, \beta$ intersect the same infinite sequence $X_{\alpha_{0}}, X_{\beta_{0}}, X_{\alpha_{1}}, X_{\beta_{1}}, \ldots$ of blocks. This follows from (3.4.3) and (3.4.6). Hence, $\alpha, \beta \in \mathcal{I}_{w}$.

Furthermore, by (3.4.7) we have

$$
\left|\alpha\left(t_{2 i}\right) \alpha_{i}\left(t_{2 i}\right)\right| \leq \epsilon \quad \text { and } \quad\left|\beta\left(t_{2 i}\right) \beta_{i}\left(t_{2 i}\right)\right| \leq \epsilon
$$

for every $i \geq 1$. Together with (3.4.5) this gives

$$
\angle \bar{\alpha}\left(t_{2 i}\right) \bar{x}_{0} \bar{\beta}\left(t_{2 i}\right) \geq \omega_{u}-\epsilon_{2 i}-\epsilon / t_{2 i} .
$$

Hence, $\angle(\alpha, \beta) \geq \omega_{u}$ and by Remark 3.2.1, $L(w)=\omega_{u}$.

\section{The length spectrum of primitive components}

Theorem 0.4 can be reformulated as follows.

3.5. Theorem. Assume that for a gluing torus $T_{u} \subset M$ the angle between the $S^{1}$-factors of the adjacents maximal blocks of $M$ (which can coincide) is $\omega_{u}=\pi / 2$. Then given $l \in\left[0, \omega_{u}\right]$ there exists a nonstandard component $\mathcal{I}_{w}, w \in \mathcal{W}_{u}$ with the length $L(w)=l$.

Theorem 3.5 is already proved for $l=\omega_{u}$ in Theorem 0.2 even without the condition $\omega_{u}=\pi / 2$. Thus in the sequel, we assume that $0 \leq l<\omega_{u}$. This is essential for the proof, because it uses an approach distinct from that of Theorem 0.2, which does not work for $l=\omega_{u}$. The key step in the proof is Proposition 3.6 below (the Collapsing Proposition). The restriction $\omega_{u}=\pi / 2$ is basically related to it.

Recall that a pair $(\alpha, \beta)$ of neighboring singular points of $\mathcal{F}$ belongs to the class $\mathcal{W}_{u}$, if the separating flat $E_{\alpha \beta}$ covers the torus $T_{u}$ and $\angle(\alpha, \beta)=\omega_{u}$. The flat $E_{\alpha \beta}$ is also called $(\alpha, \beta)$-window. 
Fix $x_{0} \in X$ and consider an (ordered) pair $(\beta, \alpha)$ of the class $\mathcal{W}_{u}$ so that $\beta<\alpha$ w.r.t. $x_{0}$. Let $R>0$. The $(\beta, \alpha)$-window is located in a $R$-restricted direction, if $\left|x_{0} x_{\alpha}\right| \leq R$, where the ray $c_{\alpha}$ in $X$ emanating from $x_{0}$ intersects $E_{\alpha \beta}$ orthogonally and $x_{\alpha} \in c_{\alpha} \cap X_{\beta}$ is the entering point of $c_{\alpha}$ into the block $X_{\beta}$.

Furthermore, the $(\beta, \alpha)$-window is said to be $\tau$-thin, $\tau>0$, if $\underline{\alpha}, \underline{\alpha}^{\prime} \in U_{x_{\alpha}, 10 \tau}\left(c_{\alpha}\right)$, where $x_{\alpha}, c_{\alpha}$ are as above, $\underline{\alpha}, \underline{\alpha}^{\prime}$ are the midpoints of the circle $S_{\alpha \beta}=\partial_{\infty} E_{\alpha \beta}$ between $\beta$ and $-\beta$ (in the case of a $\pi / 2$-metric, $\underline{\alpha}=\alpha, \underline{\alpha}^{\prime}=-\alpha$ ).

Of course, it is assumed that then $\tau$ is larger, the window is thinner.

Recall that

$$
U_{x_{0}, t}(z)=\left\{z^{\prime} \in \partial_{\infty} X|| z(t) z^{\prime}(t) \mid<1\right\}, \quad z \in \partial_{\infty} X, t>0,
$$

is a neighborhood of $z \in \partial_{\infty} X$, where a point $z^{\prime} \in \partial_{\infty} X$ is identified with the ray $z^{\prime}:[0, \infty) \rightarrow X, z^{\prime}(0)=x_{0}, z^{\prime}(\infty)=z^{\prime}$.

3.6. Proposition. Assume that $\omega_{u}=\pi / 2$. Given $x_{0} \in X$, a pair $\left(\alpha_{0}, \beta_{0}\right)$ of the class $\mathcal{W}_{u}, \alpha_{0}<\beta_{0}$ w.r.t. $x_{0}, s \in\left[\omega_{u} / 2, \omega_{u}\right), R, t>0$, then there exists $\tau>0$ such that if for $\alpha>\beta_{0}$ the $\left(\beta_{0}, \alpha\right)$-window belongs to the class $\mathcal{W}_{u}$, is $\tau$-thin and located in a $R$-restricted direction, then for any $\rho>0$ there is a singular point $\beta^{\prime} \in \mathcal{F}$ with

(i) $\beta^{\prime}>\alpha$ and $\left(\alpha, \beta^{\prime}\right)$ belongs to the class $\mathcal{W}_{u}$;

(ii) the $\left(\alpha, \beta^{\prime}\right)$-window is $\rho$-thin and located in a $R_{\alpha}$-restricted direction, where $R_{\alpha}>0$ depends only on $\alpha$;

(iii) if $b_{\alpha} \in \alpha \beta_{0}$ is the point with $\angle\left(\alpha, b_{\alpha}\right)=s$ and $b_{\alpha}^{\prime} \in \alpha \beta^{\prime}$ is the point with $\angle\left(\alpha, b_{\alpha}^{\prime}\right)=s$, then the segment $\beta^{\prime} b_{\alpha}^{\prime} \subset U_{x_{0}, t}\left(b_{\alpha}\right)$;

(iv) $a^{\prime} \in U_{x_{0}, t}(a)$ for any $0 \leq s^{\prime} \leq s$, where $a \in \alpha \beta_{0}, a^{\prime} \in \alpha \beta^{\prime}$ with $\angle(\alpha, a)=s^{\prime}=$ $\angle\left(\alpha, a^{\prime}\right)$.

The properties (iii), (iv) are the most important ones for constructing a component $w \in \mathcal{W}_{u}$ with prescribed length. They mean, roughly, that the subsegment $b_{\alpha}^{\prime} \beta^{\prime} \subset \alpha \beta^{\prime}$ collapses when viewing from $x_{0}$ (while $\alpha b_{\alpha}^{\prime}$ is observed almost at the given angle $s$ ). For this reason we refer to Proposition 3.6 as to the Collapsing Proposition.

We start the proof of Proposition 3.6 with the following

3.6.1. Lemma. Under the condition of Proposition 3.6, there exists $\tau^{\prime}>0$ such that if for $\alpha>\beta_{0}$ the $\left(\beta_{0}, \alpha\right)$-window belongs to the class $\mathcal{W}_{u}$, is $\tau^{\prime}$-thin, located in a $R$-restricted direction and $a \in \alpha \beta_{0}, \angle(\alpha, a)=s^{\prime}, 0 \leq s^{\prime} \leq s$, then

$$
U_{x_{\alpha}, \underline{\tau}^{\prime}}(a) \subset U_{x_{0}, 2 t}(a)
$$

for $\underline{\tau}^{\prime}=\tau^{\prime} / \cos s^{\prime}$, where $x_{\alpha} \in \partial X_{\beta_{0}}$ as in the definition of a $\tau$-thin window.

Proof. If $x_{0} \in X_{\beta_{0}}$, then $x_{\alpha}=x_{0}$, and the lemma is trivial. Thus we suppose that $x_{0} \notin X_{\beta_{0}}$. In that case the entering points $x_{\alpha}$ 's into the block $X_{\beta_{0}}$ are contained in a compact subset $K$ of the corresponding boundary component of $X_{\beta_{0}}$ by the condition of the $R$-restricted direction. Assuming that the lemma is not true and using the condition $s^{\prime} \leq s<\pi / 2$, we find corresponding limit points $a_{\infty} \in \partial_{\infty} X_{\beta_{0}}$, $x_{\infty} \in K$, for which a required neighborhood exists by properties of the standard topology. This is incompatible with our assumption.

In the sequel, we use notation $[x y\rangle$ for the ray in $X$ emanating from $x \in X$ and passing through $y \in X \cup \partial_{\infty} X$. 
Proof of Proposition 3.6. We pick $\tau^{\prime}>0$ provided by Lemma 3.6.1 and take $\epsilon>0$ with $\tan s-\tan (s-\epsilon)=1 / 4 \tau^{\prime}$. In other words, if $x y z \subset \mathbb{R}^{2}$ is a triangle with $|x y|=\tau^{\prime}, \angle x y z=\pi / 2, \angle y x z=s$, and $h \in y z$ satisfies $|h z|=1 / 4$, then $\angle z x h=\epsilon$. Notice that $\epsilon \rightarrow 0$ as $s$ is fixed and $\tau^{\prime} \rightarrow \infty$. In particular, we assume that $\epsilon<s$, $\pi / 2-s$.

Since the factors $Y_{\alpha}$ of the decompositions $X_{\alpha}=Y_{\alpha} \times \mathbb{R}, \alpha \in \mathcal{A}$ are hyperbolic (see 2.2.3), there is $H>0$ such that if $|p q| \geq H / 16$ for an infinite triangle $p q r$ in $Y_{\alpha}, p, q \in Y_{\alpha}, r \in \partial_{\infty} Y_{\alpha}, \angle p q r=\pi / 2$, then $\angle q p r \leq \epsilon^{3}$. Now we take $\tau=H \cdot \tau^{\prime}$.

Step 1: choosing a disc $D_{\alpha}$. Let $\alpha \in \mathcal{F}$ be a singular point such that $\alpha>\beta_{0}$ w.r.t. $x_{0},\left(\beta_{0}, \alpha\right)$-window belongs to the class $\mathcal{W}_{u}$, is $\tau$-thin and let $b_{\alpha} \in \alpha \beta_{0}$ with $\angle\left(\alpha, b_{\alpha}\right)=s$. For the ray $c_{\alpha}$ in $X$, which emanates from $x_{0}$ and meets the window $E_{\alpha \beta_{0}}$ orthogonally at $y_{\alpha}$ let $x_{\alpha} \in c_{\alpha} \cap X_{\beta_{0}}$ be the entering point into $X_{\beta_{0}}$.

We take $d_{\alpha} \in E_{\alpha \beta_{0}}$ with $L_{x_{\alpha}}\left(d_{\alpha}, y_{\alpha}\right)=s$, which projects along the $\mathbb{R}$-factor of $X_{\beta_{0}}$ in $y_{\alpha}$. Let $D_{\alpha} \subset E_{\alpha \beta_{0}}$ be the disc of radius $H_{\alpha}=H \cdot\left|x_{\alpha} y_{\alpha}\right| / 2 \tau$ centered at $d_{\alpha}$ (since $\left(\beta_{0}, \alpha\right)$-window is $\tau$-thin, we have $\left|x_{\alpha} y_{\alpha}\right|>\tau$ ).

Let $d_{\alpha}^{ \pm} \in D_{\alpha}$ be the ends of the vertical diameter of $D_{\alpha}$, i.e. the segment $d_{\alpha}^{-} d_{\alpha}^{+}$ is parallel to the $\mathbb{R}$-factor of $X_{\beta_{0}}$. We assume that $d_{\alpha}^{-}$lies between $d_{\alpha}$ and $y_{\alpha}$. Then by the choice of $H_{\alpha}, \tau$ and $\tau^{\prime}$ we have $\left[x_{\alpha} d_{\alpha}^{ \pm}\right\rangle \in U_{x_{\alpha}, 2 \underline{\tau}^{\prime}}\left(\left[x_{\alpha} d_{\alpha}\right\rangle\right)$, where $\underline{\tau}^{\prime}=\tau^{\prime} / \cos s$. Moreover, $\left[x_{\alpha} x\right\rangle \in U_{x_{\alpha}, 2 \underline{\tau}^{\prime}}\left(\left[x_{\alpha} d_{\alpha}\right\rangle\right)$ for the remaining points $x \in D_{\alpha}$, since the curvature of the factor $Y_{\alpha}$ is nonpositive. Recalling the choice of $d_{\alpha}, b_{\alpha}$ and the definition of a $\tau$-thin window, we obtain that

$$
U_{x_{\alpha}, 2{\underline{\tau^{\prime}}}^{\prime}}\left(\left[x_{\alpha} d_{\alpha}\right\rangle\right) \subset U_{x_{\alpha}, \underline{\underline{\tau}}^{\prime}}\left(b_{\alpha}\right) .
$$

Now by the choice of $\tau^{\prime}$ and Lemma 3.6.1, we have

$$
z_{x} \in U_{x_{0}, 2 t}\left(b_{\alpha}\right)
$$

for any $x \in D_{\alpha}$, where $z_{x}=\left[x_{\alpha} x\right\rangle(\infty)$.

The last property allows one to replace the point of view from $x_{0}$ to $x_{\alpha}$ while considering a $\tau$-thin window $E_{\alpha \beta_{0}}$. The advantage is that the initial segments $x_{\alpha} x$ of rays $\left[x_{\alpha} x\right\rangle, x \in D_{\alpha}$ are contained in the block $X_{\beta_{0}}$, and we can use the splitting $X_{\beta_{0}}=Y_{\beta_{0}} \times \mathbb{R}$ to make necessary calculations.

Step 2: choosing $\beta^{\prime}$. Let $h_{\alpha}$ be the midpoint of $d_{\alpha}^{-} d_{\alpha}, J_{\alpha}=\left[h_{\alpha}-\rho_{1}, h_{\alpha}\right]$ the subsegment of $d_{\alpha}^{-} h_{\alpha}$ of length $\rho_{1}$ (with obvious notation), where the constant $\rho_{1}$ is provided by Lemma 2.2.5. Since $H_{\alpha} \rightarrow \infty$ as $\tau \rightarrow \infty$, while $\rho_{1}$ is independent of any choice made in the proposition, we can assume that $\rho_{1} \leq H_{\alpha} / 8$.

Since $\omega_{u}=\pi / 2$, the interval $X_{\alpha} \cap\left[x_{\alpha} x\right\rangle$ lies in a horizontal slice $Y_{\alpha} \times\{\mathrm{pt}\}$ of $X_{\alpha}=Y_{\alpha} \times \mathbb{R}$ for any $x \in d_{\alpha}^{-} d_{\alpha}^{+}$(here we use the fact that the ray $c_{\alpha}$ meets $E_{\alpha \beta_{0}}$ orthogonally). Hence, by the periodicity argument, there are points $d, d^{\prime} \in J_{\alpha}$ such that the segments $X_{\alpha} \cap\left[x_{\alpha} d\right\rangle, X_{\alpha} \cap\left[x_{\alpha} d^{\prime}\right\rangle$ connect $E_{\alpha \beta_{0}}$ with different boundary components of $X_{\alpha}$. It immediately follows that for any $\rho>0$ we can find a $\rho$ thin window $E_{\alpha \beta^{\prime}}$ in the corridor $\left(d d^{\prime}\right) \subset J_{\alpha}$ (i.e. the rays $\left[x_{\alpha} \underline{\beta}\right\rangle,\left[x_{\alpha} \underline{\beta^{\prime}}\right\rangle$ intersect $E_{\alpha \beta_{0}}$ in $\left(d d^{\prime}\right)$, where $\underline{\beta}=\beta^{\prime}, \underline{\beta}^{\prime}=-\beta^{\prime} \in S_{\alpha \beta^{\prime}}$ are the midpoints between $\alpha$ and $-\alpha)$ with $\beta^{\prime}>\alpha$ w.r.t. $x_{0}$, the pair $\left(\alpha, \beta^{\prime}\right)$ belongs to the class $\mathcal{W}_{u}$ and $E_{\alpha \beta^{\prime}}$ is located in a $R_{\alpha}$-restricted direction, where $R_{\alpha}$ depends only on $\alpha$. Therefore, the properties (i), (ii) of the proposition are proved. Moreover, it follows from (3.6.2) that $\beta^{\prime} \in U_{x_{0}, 2 t}\left(b_{\alpha}\right)$. 
Step 3: shifting vertically. The rays $\left[x_{\alpha} d_{\alpha}\right\rangle,\left[x_{\alpha} h_{\alpha}\right\rangle$ cut out a segment of length $\tau^{\prime} \cdot H_{\alpha} / 2\left|x_{\alpha} y_{\alpha}\right|=\tau^{\prime} \cdot H / 4 \tau=1 / 4$ on the vertical line $\mathbb{R} \times\{\mathrm{pt}\} \subset X_{\beta_{0}}$ projecting in the point $\left[x_{\alpha} y_{\alpha}\right\rangle\left(\tau^{\prime}\right)$. Then by the choice of $\epsilon$ we have $\angle_{x_{\alpha}}\left(d_{\alpha}, h_{\alpha}\right)=\epsilon$.

Consider the points $h_{\alpha}^{\prime}=\left[x_{\alpha} \beta^{\prime}\right\rangle \cap J_{\alpha}$ and $h_{\alpha}^{\prime \prime} \in d_{\alpha}^{-} h_{\alpha}$ with $\left|h_{\alpha} h_{\alpha}^{\prime \prime}\right|=H_{\alpha} / 4$. By the choice of $H_{\alpha}>H / 2$ and $H$ we see that $h_{\alpha}^{\prime \prime}$ is below of the interval $J_{\alpha}$ and $\left|h_{\alpha}^{\prime} h_{\alpha}^{\prime \prime}\right| \geq H / 16$.

The infinite triangle $h_{\alpha}^{\prime \prime} h_{\alpha}^{\prime} \beta^{\prime} \subset Y_{\alpha} \times\{$ pt $\}, \beta^{\prime} \in \partial_{\infty} Y_{\alpha}$ has the angle $\angle h_{\alpha}^{\prime \prime} h_{\alpha}^{\prime} \beta^{\prime} \geq$ $\pi / 2$. Hence $\angle h_{\alpha}^{\prime} h_{\alpha}^{\prime \prime} \beta^{\prime} \leq \epsilon^{3}$ by the choice of $H$. On the other hand, $\angle d_{\alpha} h_{\alpha}^{\prime} \beta^{\prime} \geq$ $\angle_{x_{\alpha}}\left(d_{\alpha}, h_{\alpha}\right)=\epsilon$. Thus there exists $h_{0} \in\left(h_{\alpha}^{\prime \prime} h_{\alpha}^{\prime}\right)$ with

$$
\tan \bar{\psi}_{0}=\frac{2}{\pi} \epsilon^{2}
$$

for $\bar{\psi}_{0}=\angle h_{\alpha}^{\prime} h_{0} \beta^{\prime}$.

Step 4: shifting horizontally. On the ray $\left[h_{0} \alpha\right\rangle \subset E_{\alpha \beta_{0}}$ we consider the segment $h_{0} h_{1}$ of length $r_{1}=H_{\alpha} / 8$. Then $h_{0} h_{1} \subset D_{\alpha}$. We denote by $h_{r} \in h_{0} h_{1}$ the point with $\left|h_{0} h_{r}\right|=r \cdot r_{1}$ for $0 \leq r \leq 1$.

On the unit sphere of the tangent space $T_{h_{r}} X$ consider the outgoing direction $v(r)$ of the segment $x_{\alpha} h_{r}$ and its projections $v_{\alpha}(r)$ on $Y_{\alpha}$ and $v_{\beta_{0}}(r)$ on $Y_{\beta_{0}}$ (in the obvious sense). We denote by $\phi_{r}=\angle\left(v_{\beta_{0}}(r), \alpha\right), \psi_{r}=\angle\left(v_{\alpha}(r), \beta_{0}\right)$ and $\gamma_{r}=\angle\left(v(r), \beta_{0}\right)$. Clearly, $\gamma_{r} \geq \gamma_{0} \geq \pi / 2-(s-\epsilon) \geq \epsilon$ for each $0 \leq r \leq 1$.

Using the facts that $\left[x_{\alpha} h_{r}\right\rangle(\infty) \in U_{x_{0}, 2 t}\left(b_{\alpha}\right)$ by $(3.6 .2)$ and $s \geq \omega_{u} / 2$, one can easily see that $\pi / 2-\gamma_{r}$ is separated from 0 by a constant $c>0$ independently of $t \rightarrow \infty$. We can assume that $\epsilon^{3} / c<2 \epsilon^{2} / \pi$.

A simple exercise in the spherical trigonometry shows that

$$
\sin \phi_{r}=\tan \psi_{r} / \tan \gamma_{r}, \quad 0 \leq r \leq 1
$$

Since $\left|h_{0} h_{1}\right|=H_{\alpha} / 8$, it follows from hyperbolicity of $Y_{\beta_{0}}$ that $\phi_{1} \leq \epsilon^{3}$ by the choice of $H$. Thus

$$
\tan \psi_{1}=\sin \phi_{1} \tan \gamma_{1} \leq \epsilon^{3} / c<2 \epsilon^{2} / \pi
$$

Hence, we can find $r \in(0,1)$ with $\psi_{r}=\bar{\psi}_{0}$, i.e. $\tan \psi_{r}=\tan \bar{\psi}_{0}=2 \epsilon^{2} / \pi$, because $\psi_{0}=\angle h_{0} x_{\alpha} \beta_{0} \geq \epsilon$.

This implies that the part of the ray $\left[x_{\alpha} h_{r}\right\rangle$ which lies in $X_{\alpha}$ projects onto the ray $\left[h_{0} \beta^{\prime}\right\rangle$ in the corresponding slice $Y_{\alpha} \times\{\mathrm{pt}\}$. Therefore we have $b_{\alpha}^{\prime \prime}=\left[x_{\alpha} h_{r}\right\rangle(\infty) \in$ $\alpha \beta^{\prime}$. For such $r$ we have

$$
\frac{2}{\pi} \phi_{r} \leq \sin \phi_{r} \leq \frac{1}{\epsilon} \tan \psi_{r}
$$

because $\gamma_{r} \geq \epsilon$. Hence, $\phi_{r} \leq \epsilon$.

The spherical triangle $v(r) v_{\beta_{0}}(r) \alpha$ has a right angle at the vertex $v_{\beta_{0}}$. It follows that

$$
(\angle(\alpha, v(r)))^{2} \leq \phi_{r}^{2}+\left(\pi / 2-\gamma_{r}\right)^{2} \leq \epsilon^{2}+(s-\epsilon)^{2}<s^{2}
$$

by the choice of $\epsilon$.

Using the splitting $X_{\alpha}=Y_{\alpha} \times \mathbb{R}$, we obtain that $\angle(\alpha, v(r))=\angle\left(\alpha, b_{\alpha}^{\prime \prime}\right)$. Therefore, we have found the point $b_{\alpha}^{\prime \prime}$ on the segment $\alpha \beta^{\prime} \subset \partial_{T} X$, for which $\angle\left(\alpha, b_{\alpha}^{\prime \prime}\right)<s$ and $b_{\alpha}^{\prime \prime} \in U_{x_{0}, 2 t}\left(b_{\alpha}\right)$ by (3.6.2). Since $\angle\left(\alpha, b_{\alpha}^{\prime}\right)=s$ and $\beta^{\prime} \in U_{x_{0}, 2 t}\left(b_{\alpha}\right)$, an easy argument shows that $\beta^{\prime} b_{\alpha}^{\prime} \subset U_{x_{0}, 2 t}\left(b_{\alpha}\right) \subset U_{x_{0}, t}\left(b_{\alpha}\right)$. This gives (iii). 
It remains to prove (iv). For $z \in \partial_{T} X$ let $\xi(z)$ be the angle between the ray $\left[x_{\alpha} z\right\rangle$ and a horizontal slice $Y_{\beta_{0}} \times\{\mathrm{pt}\}, \xi(z)=\pi / 2-\angle_{x_{\alpha}}\left(\beta_{0}, z\right)$. Then we have $\xi\left(a^{\prime}\right) \leq \xi(a)=s^{\prime}, \xi\left(b_{\alpha}^{\prime}\right) \leq \xi\left(b_{\alpha}\right)=s$ and $\xi\left(b_{\alpha}^{\prime}\right)-\xi\left(a^{\prime}\right) \leq s-s^{\prime}$.

If the claim is false, then $\xi(a)-\xi\left(a^{\prime}\right)>\xi\left(b_{\alpha}\right)-\xi\left(b_{\alpha}^{\prime}\right)$. This follows from Lemma 3.6.1, the already proved condition $b_{\alpha}^{\prime} \in U_{x_{0}, 2 t}\left(b_{\alpha}\right) \subset U_{x_{0}, t}\left(b_{\alpha}\right)$ and that the horizontal projections of the considered rays emanating from $x_{\alpha}$ diverge negligibly small when compared to their vertical divergence while $t$ is fixed, because $\left(\beta_{0}, \alpha\right)$-window is $\tau$-thin. Thus

$$
s-s^{\prime} \geq \xi\left(b_{\alpha}^{\prime}\right)-\xi\left(a^{\prime}\right)=\xi\left(b_{\alpha}\right)-\xi(a)+\xi(a)-\xi\left(a^{\prime}\right)-\left(\xi\left(b_{\alpha}\right)-\xi\left(b_{\alpha}^{\prime}\right)\right)>s-s^{\prime},
$$

a contradiction. This completes the proof of Proposition 3.6.

3.7. Lemma. Given $x_{0} \in X$, a pair $\left(\alpha_{0}, \beta_{0}\right)$ of the class $\mathcal{W}_{u}, \alpha_{0}<\beta_{0}$ w.r.t. $x_{0}$, $s \in\left[\omega_{u} / 2, \omega_{u}\right), R, \epsilon>0$, there exists $\tau>0$ such that if for $\alpha>\beta_{0}$ the $\left(\beta_{0}, \alpha\right)$ window belongs to the class $\mathcal{W}_{u}$, is $\tau$-thin and located in an $R$-restricted direction, and $a_{\alpha}, b_{\alpha} \in \alpha \beta_{0}, \angle\left(\alpha, b_{\alpha}\right)=\angle\left(a_{\alpha}, \beta_{0}\right)=s$, then

$$
l-\epsilon \leq \angle \overline{a_{\alpha}}(\tau) \bar{x}_{0} \overline{b_{\alpha}}(\tau) \leq l,
$$

where $l=2 s-\omega_{u}, \overline{x_{0}} \overline{a_{\alpha}}(\tau) \overline{b_{\alpha}}(\tau) \subset \mathbb{R}^{2}$ is the comparison triangle for $x_{0} a_{\alpha}(\tau) b_{\alpha}(\tau)$ (as usual, we identify a point of $\partial_{\infty} X$ with the ray emanating from $x_{0}$ ).

Proof. The assertion is trivial, if $x_{0} \in X_{\beta_{0}}$, i.e. $R=0$, because of the splitting $X_{\beta_{0}}=Y_{\beta_{0}} \times \mathbb{R}$. Now assume that $x_{0} \notin X_{\beta_{0}}$. Let $x_{\alpha}$ be the entering point in $X_{\beta_{0}}$ of the ray $c_{\alpha}=\left[x_{0} y_{\alpha}\right\rangle$, where $y_{\alpha} \in E_{\beta_{0} \alpha}$ is the point closest to $x_{0}$. It follows from the condition of the $R$-restricted direction and the periodicity argument that the entering points of the rays $a_{\alpha}, b_{\alpha}$ into the block $X_{\beta_{0}}$ are at the distance from $x_{\alpha}$ bounded by a constant depending only on $R, \omega_{u}-s$ and $\rho_{1}$ (the constant from Lemma 2.2.5) for any $\tau$ and $\tau$-thin window $E_{\beta_{0} \alpha}$.

Thus the distances dist $\left(a_{\alpha}(\tau),\left[x_{\alpha} a_{\alpha}\right\rangle\right)$, dist $\left(b_{\alpha}(\tau),\left[x_{\alpha} b_{\alpha}\right\rangle\right)$ are bounded independently of $\tau$ and $\tau$-thin window $E_{\beta_{0} \alpha}$. Hence, the claim.

3.8. Proof of Theorem 3.5. Recall some standard notations, which will be used in the proof.

For $x_{0} \in X$ and a pair of neighboring singular points $\alpha, \beta \in \mathcal{F}, \alpha<\beta$ w.r.t. $x_{0}$ we denote $S_{\alpha \beta}=\partial_{\infty} E_{\alpha \beta}$. The complement $\partial_{\infty} X \backslash S_{\alpha \beta}$ consists of two open discs; let $\mathcal{D}_{\alpha \beta}$ be that, for which every ray $\left[x_{0} z\right\rangle, z \in \mathcal{D}_{\alpha \beta}$ meets the flat $E_{\alpha \beta}$.

For $l \in\left[0, \omega_{u}\right)$ let $s=l+\left(\omega_{u}-l\right) / 2$. Then $s \in\left[\omega_{u} / 2, \omega_{u}\right)$. We fix $\epsilon>0$, a sequence $\epsilon_{i}>0$ with $\sum_{i} \epsilon_{i} \leq \epsilon$, a point $x_{0} \in X$, a pair $\left(\alpha_{0}, \beta_{0}\right)$ of the class $\mathcal{W}_{u}$, $\alpha_{0}<\beta_{0}$ w.r.t. $x_{0}$.

Step 0. We choose two neighboring singular points $\beta_{\#}$ and $\alpha_{0}$ in $\mathcal{F}$, such that the separating flat $E_{\beta_{\#} \alpha_{0}}$ covers $T_{u}$. We choose our starting point $x_{0}$ in $E_{\beta_{\#} \alpha_{0}}$ and (by slight abuse of notation) we consider the pair $\left(\beta_{\#}, \alpha_{0}\right)$ as a pair of class $\mathcal{W}_{u}$ with $\beta_{\#}<\alpha_{0}$ which is located in an $R$-restricted direction for $R=0$. By applying the Collapsing Proposition to $x_{0},\left(\beta_{\#}, \alpha_{0}\right), s, R=0, t_{0}=1 / \epsilon_{0}$ we obtain a $\tau_{0} \geq t_{0}$ such that if a window $\left(\alpha_{0}, \beta_{0}\right)$ belongs to the class $\mathcal{W}_{u}$, is $\tau_{0}$-thin and located in an $R$-restricted direction, then for every $\rho>0$ there is a singular point $\alpha^{\prime} \in \mathcal{F}$, $\alpha^{\prime}>\beta_{0}$, for which the conditions (i)-(iv) of the Collapsing Proposition are fulfilled for $t=t_{0}$. Clearly, such a $\tau_{0}$-thin window $\left(\alpha_{0}, \beta_{0}\right)$ exists.

Let $J_{0}=a_{0} b_{0} \subset \alpha_{0} \beta_{0}$ be the middle segment of length $l$, i.e. $\angle\left(\alpha_{0}, b_{0}\right)=$ $\angle\left(a_{0}, \beta_{0}\right)=s$. 
Since $\angle\left(a_{0}, b_{0}\right)=l$ and the rays $a_{0}, b_{0}$ (emanating from $\left.x_{0}\right)$ stay in $X_{\alpha_{0}}$, we have $\angle_{x_{0}}\left(a_{0}(t), b_{0}(t)\right)=l$ for any $t>0$. We put

$$
V_{0}=\bigcup_{c \in J_{0}} U_{x_{0}, t_{0}}(c) \text {. }
$$

For any $z \in \mathcal{D}_{\alpha_{0} \beta_{0}}$ with $\left(\omega_{u}-l\right) / 2 \leq L_{x_{0}}\left(z, \alpha_{0}\right) \leq s$ we have $z \in V_{0}$, since $\left(\alpha_{0}, \beta_{0}\right)$ window is $\tau_{0}$-thin and $\tau_{0} \geq t_{0}$.

Step 1. We put $R_{1}=R_{\beta_{0}}$ (see condition (ii) of the Collapsing Proposition obtained in Step 0$), t_{1}=1 / \epsilon_{1}$, and for $x_{0},\left(\alpha_{0}, \beta_{0}\right), s, R_{1}, t_{1}$ using the Collapsing Proposition we find $\tau_{1} \geq t_{1}$ such that if for $\alpha_{1}>\beta_{0}$ the $\left(\beta_{0}, \alpha_{1}\right)$-window belongs to the class $\mathcal{W}_{u}$, is $\tau_{1}$-thin, located in an $R_{1}$-restricted direction, and $a_{\alpha_{1}}, b_{\alpha_{1}} \in \alpha_{1} \beta_{0}, \angle\left(\alpha_{1}, b_{\alpha_{1}}\right)=$ $\angle\left(a_{\alpha_{1}}, \beta_{0}\right)=s$, then for any $\rho>0$ there is a singular point $\beta^{\prime} \in \mathcal{F}, \beta^{\prime}>\alpha_{1}$, for which the conditions (i)-(iv) of the Collapsing Proposition are fulfilled for $t=t_{1}$.

By the conditions (i), (ii) of the Collapsing Proposition obtained in Step 0, such $\alpha_{1}$ does exist. Furthermore, it follows from (iii), (iv) of Step 0 that $U_{x_{0}, t_{1}}\left(a_{\alpha_{1}}\right) \subset$ $U_{x_{0}, t_{0}}\left(a_{0}\right), U_{x_{0}, t_{1}}\left(b_{\alpha_{1}}\right) \subset U_{x_{0}, t_{0}}\left(b_{0}\right)$ and $\alpha_{1},-\alpha_{1} \in U_{x_{0}, t_{0}}\left(a_{0}\right)$.

Step 2. We put $t_{2}=1 / \epsilon_{2}$, and for $x_{0},\left(\beta_{0}, \alpha_{1}\right), s, R_{\alpha_{1}}, t_{2}, \epsilon_{2}$ using the Collapsing Proposition and Lemma 3.7 we find $\tau_{2} \geq t_{2}$ such that if for $\beta_{1}>\alpha_{1}$ the $\left(\alpha_{1}, \beta_{1}\right)$ window belongs to the class $\mathcal{W}_{u}$, is $\tau_{2}$-thin, located in the $R_{\alpha_{1}}$-restricted direction, and $a_{1}, b_{1} \in \alpha_{1} \beta_{1}, \angle\left(\alpha_{1}, b_{1}\right)=\angle\left(a_{1}, \beta_{1}\right)=s$, then

$$
l-\epsilon_{2} \leq \angle \bar{a}_{1}\left(\tau_{2}\right) \bar{x}_{0} \bar{b}_{1}\left(\tau_{2}\right) \leq l
$$

and for any $\rho>0$ there is a singular point $\alpha^{\prime} \in \mathcal{F}, \alpha^{\prime}>\beta_{1}$, for which the conditions (i)-(iv) of the Collapsing Proposition are fulfilled for $t=t_{2}$.

By Step 1 , such $\beta_{1}$ does exist, and we put $J_{1}=a_{1} b_{1}$,

$$
V_{1}=\bigcup_{c \in J_{1}} U_{x_{0}, t_{2}}(c) .
$$

By the condition (iii) of the Collapsing Proposition obtained in Step 1 we have $b_{1} \beta_{1} \in U_{x_{0}, t_{1}}\left(b_{\alpha_{1}}\right) \subset U_{x_{0}, t_{0}}\left(b_{0}\right)$, and by (iv) $a_{1} \in U_{x_{0}, t_{0}}\left(a_{0}\right)$. Furthermore, we can assume by Step 1 that $\mathcal{D}_{\alpha_{1} \beta_{1}} \subset V_{0}$.

Continuing in this way, we obtain a string $w=\alpha_{0} \beta_{0} \alpha_{1} \beta_{1} \cdots \in W$, the class of which belongs to $\mathcal{W}_{u}$. For the middle segments $J_{i}=a_{i} b_{i} \subset \alpha_{i} \beta_{i}$ of length $l$, where $\angle\left(\alpha_{i}, b_{i}\right)=\angle\left(a_{i}, \beta_{i}\right)=s$, we have $a_{i+1} \in U_{x_{0}, t_{2 i}}\left(a_{i}\right), b_{i+1} \in U_{x_{0}, t_{2 i}}\left(b_{i}\right), t_{i}=1 / \epsilon_{i}$,

$$
l-\epsilon_{2 i} \leq \angle \bar{a}_{i}\left(\tau_{2 i}\right) \bar{x}_{0} \bar{b}_{i}\left(\tau_{2 i}\right) \leq l
$$

for $\tau_{i} \geq t_{i}$. Thus $a_{i} \rightarrow a, b_{i} \rightarrow b$ as $i \rightarrow \infty$ in the standard topology, and $\angle(a, b)=l$. Furthermore, $a b \subset \mathcal{I}_{w}$ by the construction.

For the neighborhoods $V_{i}=\bigcup_{c \in J_{i}} U_{x_{0}, t_{2 i}}(c)$ we have (very important point)

$$
\mathcal{D}_{\alpha_{i} \beta_{i}} \subset V_{i-1} \text {. }
$$

This implies $\mathcal{I}_{w} \subset \bigcap_{i \geq 0} V_{i}$. Therefore, it remains to show that $\bigcap_{i \geq 0} V_{i}=a b$.

Let $z \in \bigcap_{i \geq 0} V_{i}$. Then for each $i \geq 0$ there is $z_{i} \in a_{i} b_{i}$ with $z \in U_{x_{0}, t_{2 i}}\left(z_{i}\right)$ and, hence, $z_{i} \in \bar{U}_{x_{0}, t_{2 i}}(z)$. Thus $z_{i} \rightarrow z$ in the standard topology. Now we use the following obvious fact.

Assume that for a sequence of segments $a_{i} b_{i} \subset \partial_{\infty} X$ of length $\angle\left(a_{i}, b_{i}\right) \leq \omega_{u}<\pi$ their ends $a_{i}, b_{i}$ converge in the standard topology to $a, b$ and $\lim \angle\left(a_{i}, b_{i}\right)=\angle(a, b)$. Then their midpoints $c_{i} \in a_{i} b_{i}$ converge in the standard topology to the midpoint $c \in a b$. More generally, the segments $a_{i} b_{i}$ (parametrized on $[0,1]$ ) converge in the 
standard topology pointwise to the segment $a b$. This easily follows from the lower semi-continuity of the Tits distance with respect to the standard topology and that $\partial_{T} X$ contains no bigon of length $<2 \pi$.

Therefore, $z \in a b$, and the primitive component $\mathcal{I}_{w}=a b$ has the length $l$. For $l>0$ the component $\mathcal{I}_{w}$ is nonstandard. If $l=0$, then the freedom of choice given by the construction above allows us to produce uncountably many components $\mathcal{I}_{w}$ with $L(w)=0$. One of them is necessarily nonstandard, because there are only countable many standard components. This completes the proof of Theorem 3.5.

\section{REFERENCES}

[B] W. Ballmann, Lectures on spaces of nonpositive curvature, DMV Seminar, Band 25, Basel, Birkhäuser, 1995. MR 97a:53053

[BB] W. Ballmann and M. Brin, Orbihedra of nonpositive curvature, Publ. Math. IHES 82 (1995), 169-209. MR 97i:53049

[BBE] W. Ballmann, M. Brin, and P. Eberlein, Structure of manifolds of nonpositive curvature.I, Ann. of Math. 122 (1985), 171-203. MR 87c:58092a

[BGS] W. Ballmann, M. Gromov, and V. Schroeder, Manifolds of nonpositive curvature, Progress in Math. 61, Birkhäuser, Boston-Basel-Stuttgart, 1985. MR 87h:53050

[Bu] S. Buyalo, Geodesics in Hadamard spaces, Algebra i Analiz 10 (1998), 93-123. MR 99c:53034

[BK1] S. Buyalo and V. Kobel'skiu,, Geometrization of graph-manifolds I. Conformal geometrization, St. Petersburg Math. J. 7 (1996), 185-216. MR 97k:57016

[BK2] S. Buyalo and V. Kobel'skiu, Geometrization of graph-manifolds. II Isometric geometrization, St. Petersburg Math. J. 7 (1996), 387-404. MR 97k:57017

[CK] C. Croke and B. Kleiner, The geodesic flow of nonpositively curved graphmanifolds, to appear.

[E] P. Eberlein, Geometry of Nonpositively Curved Manifolds, The University of Chicago Press, Chicago, 1996. MR 98h:53002

[HS] C. Hummel and V. Schroeder, Tits geometry associated with 4-dimensional closed real-analytic manifolds of nonpositive curvature, J. Diff. Geom. 48 (1998), 531-555. MR 99j:53058

[Ka] M. Kapovich, Flat conformal structures on 3-dimensional manifolds: the existence problem.I, Siberian Math. J. 30 (1990), 712-722. MR 91b:57017

[Kl] B. Kleiner, The local structure of length spaces with curvature bounded above, Math. Z. 231 (1999), 409-456. CMP 99:16

[Le] B. Leeb, 3-manifolds with(out) metrics of nonpositive curvature, Invent. Math. 122 (1995), 277-289. MR 97g:57015

St. Petersburg Branch, Steklov Mathematical Institute, Fontanka 27, 191011 St. Petersburg, Russia

E-mail address: buyalo@pdmi.ras.ru

Institut für Mathematik, Universität ZÜrich, Winterthurer Str. 190, CH-8057 ZürICH, SWITZERLAND

E-mail address: vschroed@math.unizh.ch 